\title{
A Highly Efficient Composite Class-AB-AB Miller Op-Amp With High Gain and Stable From 15 pF Up To Very Large Capacitive Loads
}

\author{
Shirin Pourashraf ${ }^{\circledR}$, Member, IEEE, Jaime Ramirez-Angulo ${ }^{(0)}$, Fellow, IEEE, \\ Antonio J. Lopez-Martin ${ }^{\circledR}$, Senior Member, IEEE, and Ramon González-Carvajal, Senior Member, IEEE
}

\begin{abstract}
In this paper, a highly power-efficient class-AB-AB Miller op-amp is discussed. The structure uses $g_{m}$ enhancement based on local common-mode feedback to provide class-AB operation with enhanced effective $g_{m}$, open-loop gain, unity-gain frequency, and slew rate $(S R)$ without significant increase in quiescent power consumption. Utilization of a nonlinear load leads to large symmetric positive and negative $S R$ s. Stability over an extremely wide range of capacitive loads is achieved through a combination of Miller and phase-lead compensations. The unity-gain frequency does not show sensitivity to capacitive load values. A test chip prototype fabricated in $0.18-\mu \mathrm{m}$ CMOS technology shows 90.8-dB open-loop gain, 12.5-MHz bandwidth for a 25-pF load capacitance, and a factor $60 S R$ enhancement with maximum output current close to $1-\mathrm{mA}$ and $43-\mu \mathrm{A}$ total static current.
\end{abstract}

Index Terms $-C_{L}$-independent pole-zero cancelation, class-AB op-amps, $g_{m}$ boosting, nonlinear load, phase-lead compensation.

\section{INTRODUCTION}

$\mathbf{T}$ WO-STAGE (Miller) op-amps can drive resistive loads and have close to rail-to-rail output swing. One-stage opamps (OTAs) are not well suited for resistive loads since their open-loop gain $\left(A_{O L}\right)$ depends on the output resistance $\left(R_{o u t}\right)$. Besides, in order to achieve high $R_{\text {out }}$ they require cascode transistors in the output branches. This reduces output swing, which is of special concern in modern IC technology operating from reduced supply voltages. The main advantage of onestage amplifiers is that their phase margin $(P M)$ increases with increasing load capacitance $C_{L}$ and they also have symmetrical positive and negative slew rate $(S R)$. On the other hand, Miller compensated op-amps with internal dominant pole decrease their $P M$ as $C_{L}$ grows and are stable over a relatively narrow

Manuscript received October 17, 2017; revised January 18, 2018 and March 14, 2018; accepted April 18, 2018. This work was supported by the Spanish National Research Agency under Grant TEC2016-80396-C2. (Corresponding author: Shirin Pourashraf.)

S. Pourashraf and J. Ramirez-Angulo are with the VLSI Laboratory, Klipsch School of Electrical and Computer Engineering, New Mexico State University, Las Cruces, NM 88003 USA (e-mail: shirin_p@nmsu.edu; jairamir@nmsu.edu).

A. J. Lopez-Martin is with the Institute of Smart Cities, Public University of Navarra, 31006 Pamplona, Spain (e-mail: antonio.lopez@unavarra.es).

R. González-Carvajal is with the Departamento de Ingeniería Electrónica, Escuela Superior de Ingenieros, Universidad de Sevilla, E-41092 Seville, Spain (e-mail: carvajal@gte.esi.us.es).

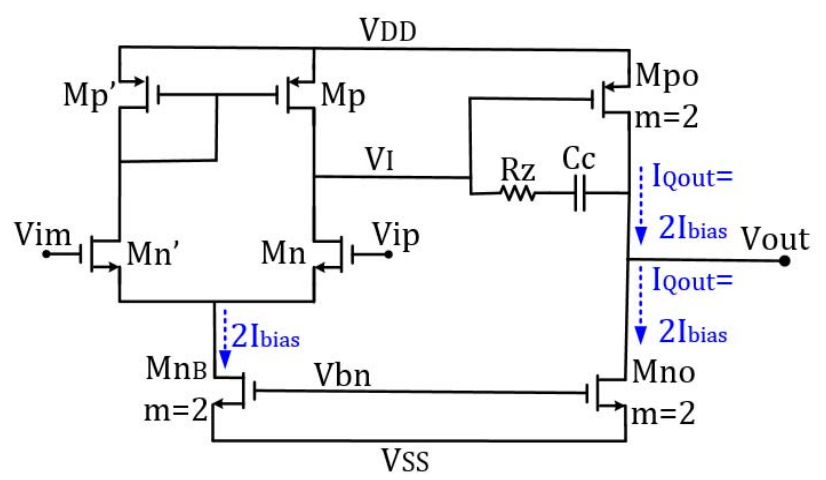

Fig. 1. Conventional two-stage class-A Miller op-amp.

range of $C_{L}$ values only. In addition, they have highly nonsymmetrical $S R$. In a conventional class-A two-stage Miller op-amp (Fig. 1) with nMOS (pMOS) input stage, negative $S R^{-}\left(S R^{+}\right)$is in general limited to an approximate value $S R^{-}=I_{\text {Qout }} /\left(C_{L}+C_{c}\right)=2 I_{\text {bias }} /\left(C_{L}+C_{c}\right)$, where $C_{c}$ is the Miller compensation capacitor and $I_{\text {bias }}$ and $I_{Q o u t}$ are the bias current of the unit transistors and the output branch, respectively. Increasing $I_{Q o u t}$ can increase $S R$ at the expense of higher static power consumption.

Class-AB op-amps [1]-[21] can deliver maximum output currents which are higher than $I_{Q o u t}$ without increasing essentially the static power consumption. They can be compared using $C E=I_{\text {outMAX }} / I_{\text {totbias }}$, where $C E$ is the current efficiency and $I_{\text {totbias }}$ is the total op-amp quiescent current. In most class-AB Miller op-amps, the gain bandwidth $(G B)$ product is determined by the transconductance gain $g_{m D P}$ of the differential input stage according to the well-known expression $G B=g_{m D P} /\left(2 \pi C_{c}\right)$. In order to increase $G B$ for a given $C_{c}$ it is required to increase the quiescent current. This results again in increased power consumption. Given that to increase the speed of an op-amp it is required to increase simultaneously $S R$ and bandwidth $(B W)$ at similar rates, a global FOM $\left(F O M_{G}\right)$ can be defined as $F O M_{G}=\left(F O M_{L} F O M_{S}\right)^{1 / 2}$. Terms $F O M_{L}=$ $I_{\text {outMAX }} / P_{\text {diss }}$ and $F O M_{S}=\left(G B C_{L}\right) / P_{\text {diss }}$ [22] are the commonly used large and small-signal $F O M \mathrm{~s}$, respectively, where $P_{\text {diss }}$ is the total power dissipation.

A simple technique with adaptive load is used in [1] to achieve class- $\mathrm{AB}$ operation; however, a high $C E$ is not 
achieved $(C E \sim 1.58)$. This is because the maximum output current depends on the maximum source-gate voltage of the output pMOS transistor which cannot go close to lower rail $V_{S S}$. In addition, $G B=g_{m D P} /\left(\pi C_{c}\right)$ has only a factor of two increase compared to the $G B$ of the conventional Miller op-amp. These reduce both $F O M_{L}$ and $F O M_{S}$. The structure in [2] has the same problem of low $C E(S R)$. This is due to the fact that it uses current starved CMOS inverters as output stages. From the point of view of $G B$, scheme in [2] performs as a conventional two-stage Miller op-amp without $g_{m}, A_{O L D C}$, and $G B$ enhancement. It also does not provide a high $P M$. The op-amp in [3] has class-AB operation while focusing on lowering the supply voltage using the body-driven (BD) technique [3], [23]-[25]. In BD op-amps, the bulk transconductance $\left(g_{m b}\right)$ is usually a factor 4-5 lower than the gate transconductance $\left(g_{m}\right)$. This degrades the $G B$, dc open-loop gain $\left(A_{O L D C}\right)$, and also leads to higher input-referred noise, offset, etc. In addition, for relatively moderate swings a p-n junction in the well of the input transistors can turn $\mathrm{ON}$ and increase significantly the input leakage current. The class-AB circuit in [5] has the ability of driving moderately high capacitive loads but its stability is provided by three compensation capacitors and resistors which increase essentially the complexity and area requirements. In addition, $C E$ does not have a high value $(C E=6.4)$ for the same reason as in [1]. Another disadvantage of [1]-[3] and [5] is that they are not capable to drive a very wide range of capacitive loads which is rather important in applications such as peak detectors, microelectromechanical systems (MEMS), liquid-crystal displays (LCDs), and line drivers [20], [21]. The class-AB scheme in [4] is a one-stage operational transconductance amplifier that uses local common mode feedback (LCMFB) to increase the $g_{m}$, the $B W, S R$, and $I_{\text {Out } M A X}$. The structure uses a phase-lead compensation resistor at the output to provide the stability over a wide range of $C_{L}$ loads [26]. However, the pole-zero cancelation provided by phase-lead compensation, only holds over a limited range of $C_{L}$ loads which results in long settling times [27]. To achieve a high gain, output transistors in [4] are cascoded, though this cannot be used in submicrometer technologies due to the low supplies. On the other hand, cascode output transistors reduce the swing of output, $S R$, and the $I_{\text {out } M A X}$. The maximum output current is also limited by the phase-lead compensation resistor.

In this paper, a composite class- $\mathrm{AB}-\mathrm{AB}$ Miller op-amp is presented that has high $C E(S R)$, essentially improved $B W$ and $A_{O L D C}$ (both over factor 10), and the ability to drive a very wide range (over four decades) of capacitive loads starting from relatively low $C_{L}$ values and maintaining a constant $f_{U G}$, high $P M$ approximately insensitive settling time to $C_{L}$ loads. This is achieved by using a combination of Miller and phase-lead compensations with a $C_{L}$-independent pole-zero cancelation. The op-amp uses LCMFB to boost the effective $g_{m}$, the $S R$, and the dynamic output current of the first stage. It also uses a nonlinear load to implement a pushpull output stage with large symmetrical dynamic and welldefined low quiescent output currents. The combination of LCMFB and nonlinear load results in a cumulative effect of
class-AB (denoted as class-AB-AB) leading to higher $F O M_{L}$ and $\mathrm{FOM}_{S}$ compared to other recent references. In addition, a detailed and careful design procedure is discussed.

The rest of this paper is organized as follows. Section II describes the proposed class-AB-AB design. Simulation and experimental results of a test chip and a comparison with other recent class-AB structures are discussed in Section III. Conclusions are drawn in Section IV.

\section{Fundamentals of the Proposed Circuit}

\section{A. Circuit Operation}

Fig. 2(a) and (b) shows the architecture and transistor level implementation of the proposed op-amp, respectively. The first stage is a composite stage that consists of a differential pair with resistive LCMFB followed by a shell formed by transistors $\mathrm{Mp} 1, \mathrm{Mp1}^{\prime}, \mathrm{Mn} 1$, and $\mathrm{Mn}^{\prime}$.

Assuming $\left(r_{O M p} \| r_{O M n}\right) \gg R$, the small-signal differential voltage $\left(V_{X}-V_{X}^{\prime}\right)$ can be expressed by

$$
V_{X}-V_{X}^{\prime}=A_{X}\left(V_{i p}-V_{i m}\right)=A_{X} V_{i} \approx g_{m D P} R V_{i}
$$

where $V_{i}$ is the differential input voltage. The output current of the first stage is given by

$$
I_{\text {out I }}=A_{X} g_{m p 1} V_{i}=g_{m e f f} V_{i}
$$

where $g_{m e f f}=A_{x} g_{m p 1}$ is the effective transconductance of the composite first stage. The gain $A_{I}$ of the input stage is expressed by

$$
A_{I}=g_{m e f f} R_{O I}=g_{m e f f} r_{o M p 1} \| r_{o M n 1}
$$

where $R_{O I}=r_{O M p 1} \| r_{O M n 1}$ is the output resistance of the composite first stage. It can be seen that the input stage has an effective transconductance and gain which are boosted by the factor $A_{X}$. In addition, due to the linear load $R$, the maximum voltage variations $\left(V_{X}-V_{X}^{\prime}\right)_{M A X} \approx 2 I_{\text {bias }} R$ lead to peak currents in the shell transistors that can be much larger than $I_{\text {bias }}$. Therefore, the first stage shows class-AB operation since it can provide a maximum current $I_{\text {outIMAX }} \gg 2 I_{\text {bias }}$ at node $V_{I}$. This, as opposed to a conventional differential input stage with active load that generates a maximum output current of only $2 I_{\text {bias }}$ at $V_{I}$. This can limit the $S R$ in the internal node $V_{I}$ (and of the op-amp) even if a push-pull output stage is used. The pMOS transistor Mpo of the push-pull output stage is driven by $V_{I}$ while the nMOS output transistor Mno is driven by $V_{I}^{\prime}$ which is in phase with $V_{I}$ and it is derived from $V_{X}$ through an inverting amplifier with gain $-A^{\prime}$. Amplifier $A^{\prime}$ has a quiescent output voltage $V_{I Q}^{\prime}$ that generates a quiescent current $I_{\text {Qout }}=2 I_{\text {bias }}$ in Mno equal to the current of Mpo. This sets a well-defined quiescent current in the output stage $\left(I_{\text {Qout }}\right)$. Amplifier $A^{\prime}$ uses a nonlinear load that, as explained below, provides large output impedance and dynamic output current for large input signals at node $X$ while for small input signals the gain and output impedance are low $\left(\left|A^{\prime}\right| \approx 1\right)$.

The dc open-loop gain of the op-amp is given approximately by $A_{O L D C}=A_{I} A_{I I}$ where $A_{I I}=g_{m p o} R_{O I I}$ and $R_{O I I}$ is the output resistance of the output stage. Assuming pMOS and nMOS unit transistors have the same transconductance 


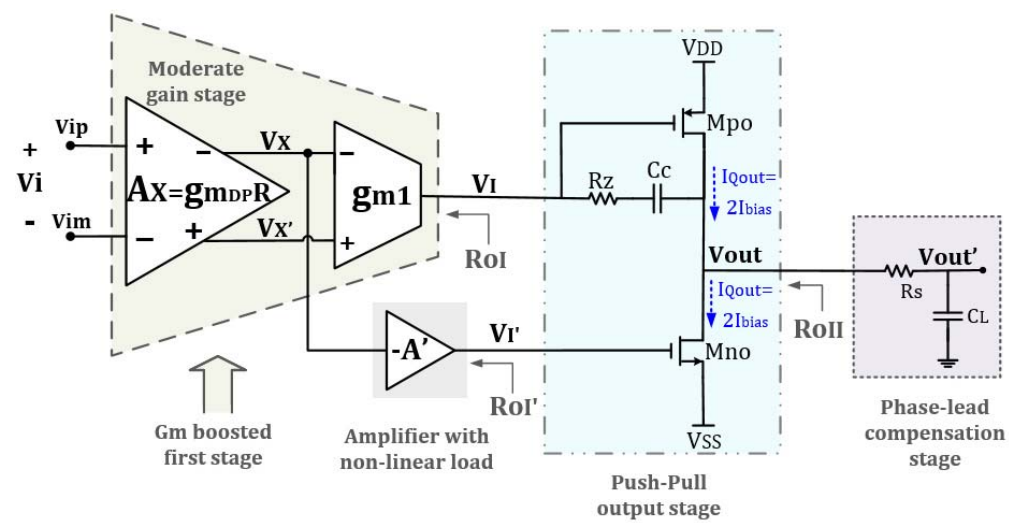

(a)

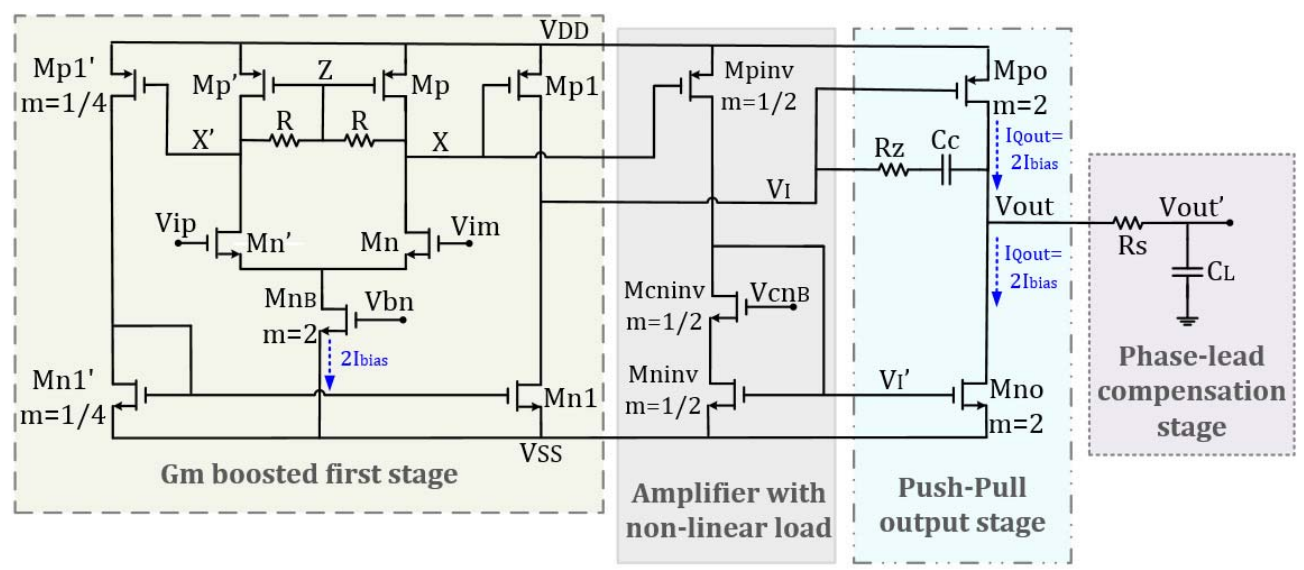

(b)

Fig. 2. (a) Architecture of the proposed class-AB-AB op-amp. (b) Transistor level implementation.

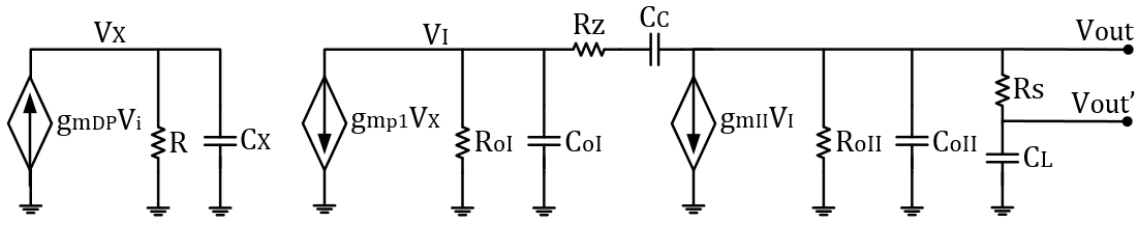

Fig. 3. Simplified small-signal equivalent circuit of the amplifier of Fig. 2.

gain $g_{m}$, the open-loop gain can be expressed by the following equation:

$$
A_{O L D C}=g_{m e f f} R_{o I} g_{m I I} R_{o I I}=g_{m D P} R\left(\frac{g_{m} r_{o}}{2}\right)^{2} .
$$

It is a factor $A_{X}$ higher than the gain of a conventional two-stage op-amp. Note that the gain contribution through $A^{\prime}$ (and Mno) has been neglected since $\left|A^{\prime}\right| \approx 1$ for small signals. The sizes of transistors $\mathrm{Mp}^{\prime}$, and $\mathrm{Mn1}^{\prime}$ are scaled down by factor 4 in order to save area, and power consumption.

Note that in the proposed class-AB-AB amplifier, the $g_{m}$ boosted first stage introduces a high frequency pole at nodes $X$ and $X^{\prime}$. Therefore, in addition to Miller compensation, a phase-lead compensation resistor $R_{S}$ connected between the output $V_{\text {out }}$ and $C_{L}$ is required in order to add a left half-plane (LHP) zero to improve the $P M$ [26].

Operation of the Nonlinear Load: The cascode voltage $V_{c n B}$ is selected so that under quiescent conditions, transistor Mninv operates in saturation close to the boundary between triode and saturation. Therefore, at dc transistors Mninv and Mno operate as a current mirror. The quiescent output current in Mno is the same as the quiescent current in Mpo $\left(I_{\text {Qout }}=2 I_{\text {bias }}\right)$. For small-signal currents transistor Mninv represents a low impedance load $1 / g_{m}$ for amplifier $A^{\prime}$ which results in a magnitude of gain $\left|A^{\prime}\right| \approx 1$. For large signal currents in the nonlinear load, Mninv enters triode operation and develops large voltage variations at node $V_{I}^{\prime}$. These lead to negative output currents in Mno which can be much larger than the quiescent current.

\section{B. Small-Signal Analysis}

The simplified small-signal equivalent circuit of the amplifier of Fig. 2(b) is shown in Fig. 3. The transfer function is given by the following equation:

$$
A(s)=\frac{V_{\text {out }}}{V_{i}}=\frac{A_{O L D C}\left(1+\frac{s}{\omega_{z 1}}\right)\left(1+\frac{s}{\omega_{z 2}}\right)}{\left(1+\frac{s}{\omega_{p d}}\right)\left(1+\frac{s}{\omega_{\text {pnd } 1}}\right)\left(1+\frac{s}{\omega_{\text {pnd } 2}}\right)}
$$


This is the author's version of an article that has been published in this journal. Changes were made to this version by the publisher prior to publication.

The final version of record is available at https://doi.org/10.1109/TVLSI.2018.2830365

TABLE I

Expressions for $A_{O L D C}$, Unity-Gain Frequency, and Pole-Zeroes in the Proposed Class-AB-AB Circuit of Fig. 2

\begin{tabular}{|c|c|c|c|c|}
\hline Pole-Zeroes & General expression & $\begin{array}{c}C_{L}<<g_{m I I} R_{o I} C_{c} \\
\text { Dominant } C_{c}\end{array}$ & $\begin{array}{c}C_{L}>>g_{m I I} R_{o I} C_{c} \\
\text { Dominant } C_{L}\end{array}$ & $\begin{array}{l}\text { Changing } \\
\text { with } C_{L} ? ?\end{array}$ \\
\hline$\omega_{p d}$ & $\frac{1}{\left(g_{m I I} R_{O I} R_{o I I} C_{c}+R_{o I I} C_{L}\right)}$ & $\approx \frac{1}{g_{m I I} R_{O I} R_{O I I} C_{c}}$ & $\approx \frac{1}{R_{o I I} C_{L}}$ & Yes \\
\hline$\omega_{p n d 1}$ & $\frac{C_{L}+g_{m I I} R_{O I} C_{c}}{R_{o I}\left(1+g_{m I I} R_{s}\right) C_{c} C_{L}}$ & $\frac{g_{m I I}}{\left(1+g_{m I I} R_{s}\right) C_{L}}$ & $\frac{1}{R_{o I}\left(1+g_{m I I} R_{s}\right) C_{c}}$ & Yes \\
\hline$\omega_{p n d 2}$ & $\frac{1}{\left(R \| R_{o I}\right) C_{X}} \approx \frac{1}{R C_{X}}$ & $\approx \frac{1}{R C_{X}}$ & $\approx \frac{1}{R C_{X}}$ & No \\
\hline$\omega_{z 1}$ & $\frac{1}{R_{S} C_{L}}$ & $\frac{1}{R_{S} C_{L}}$ & $\frac{1}{R_{S} C_{L}}$ & Yes \\
\hline$\omega_{z 2}$ & $\frac{1}{\left(R_{z}-\frac{1}{g_{m I I}}\right) C_{c}}$ & $\frac{1}{\left(R_{z}-\frac{1}{g_{m I I}}\right) C_{c}}$ & $\frac{1}{\left(R_{z}-\frac{1}{g_{m I I}}\right) C_{c}}$ & No \\
\hline$A_{O L D C}$ & $\begin{array}{c}g_{m e f f} R_{O I} g_{m I I} R_{o I I}= \\
g_{m D P} R\left(\frac{g_{m} r_{o}}{2}\right)^{2}\end{array}$ & $\begin{array}{c}g_{m e f f} R_{o I} g_{m I I} R_{o I I}= \\
g_{m D P} R\left(\frac{g_{m} r_{o}}{2}\right)^{2}\end{array}$ & $\begin{array}{c}g_{m e f f} R_{o I} g_{m I I} R_{o I I}= \\
g_{m D P} R\left(\frac{g_{m} r_{o}}{2}\right)^{2}\end{array}$ & No \\
\hline$\omega_{U G}$ & $\frac{g_{m e f f} g_{m I I} R_{s}}{C_{c}\left(1+g_{m I I} R_{s}\right)}$ & $\frac{g_{m e f f} g_{m I I} R_{s}}{C_{c}\left(1+g_{m I I} R_{s}\right)}$ & $\frac{g_{m e f f} g_{m I I} R_{s}}{C_{c}\left(1+g_{m I I} R_{s}\right)}$ & No \\
\hline
\end{tabular}

It has a dominant pole $\omega_{p d}$, two nondominant poles $\omega_{p n d} 1$, $\omega_{\text {pnd } 2}$, and two LHP zeroes $\omega_{z 1}, \omega_{z 2}$. The dc open-loop gain $A_{O L D C}$ is defined in (4).

Table I shows expressions for the poles and zeroes derived by assuming: $C_{o I}, C_{o I I}, C_{X} \ll C_{c}, C_{L}$, and $1 / R, 1 / R_{S}$, $1 / R_{z}, g_{m D P}, g_{m p 1}, g_{m I I} \gg 1 / R_{o I}, 1 / R_{o I I}$, where $C_{X}$ is the parasitic capacitance at node $X$. Since the zero $\omega_{z 2}$, and nondominant pole $\omega_{\text {pnd } 2}$ are independent of the load $C_{L}$, selecting $C_{c}$ or alternatively $R_{z}$ based on (6) causes the pole $\omega_{p n d 2}$ to approximately match the zero $\omega_{z 2}$

$$
C_{c}=\frac{R C_{X}}{\left(R_{z}-\left(\frac{1}{g_{m I I}}\right)\right)} \text { or alternatively } R_{z}=\left(\frac{R C_{X}}{C_{c}}\right)+\left(\frac{1}{g_{m I I}}\right) \text {. }
$$

This means that approximate pole-zero cancelation takes place under all loading conditions and in this case, the transfer function can be further simplified as follows:

$$
A(s) \approx \frac{A_{O L D C}\left(1+\frac{s}{\omega_{z 1}}\right)}{\left(1+\frac{s}{\omega_{p d}}\right)\left(1+\frac{s}{\omega_{p n d 1}}\right)} .
$$

Table I also shows simplified expressions for $f_{p d}, f_{p n d 1}$, and $f_{z 1}$ for two cases. Case (a) is for $C_{L} \ll g_{m I I} R_{O I} C_{c}$ which is denoted as dominant $C_{C}$, while case (b) is called dominant $C_{L}$ for $C_{L} \gg g_{m I I} R_{O I} C_{c}$.

In spite of the fact that the dominant pole $f_{p d}=\omega_{p d} /(2 \pi)$ changes with $C_{L}$, it is shown next that the open-loop unitygain frequency $\left(f_{U G}\right)$ is not dependent on $C_{L}$ and for a wide range of $C_{L}$ values it remains constant.

1) Case (a), Dominant $C c$ : In this case, $f_{z 1}$ is higher than $f_{\text {pnd } 1}$ [Fig. 4(a)]. Note that $f_{z 1}$ and $f_{p n d 1}$ change at the same rate, while the dominant pole $f_{p d}$ remains approximately constant with changes in $C_{L}$. The response in blue broken lines shows the equivalent one pole response obtained by reducing the open-loop gain by a factor $\triangle A_{O L}$ from $A_{O L D C}$ to a value $A_{O L D C} C^{\prime}$ where $\triangle A_{O L}$ is given by

$$
\begin{aligned}
\Delta A_{O L} & =\Delta f=\frac{f_{z 1}}{f_{p n d 1}} \\
A_{O L D C^{\prime}} & =\frac{A_{O L D C}}{\Delta A_{O L}}=\frac{A_{O L D C}}{\frac{f_{z 1}}{f_{p n d 1}}} .
\end{aligned}
$$

The equivalent dominant pole of the response in blue broken lines is $f_{p d}$. This leads to a unity-gain frequency $f_{U G}$ given by

$$
f_{U G}=A_{O L D C^{\prime}} f_{p d}=\frac{A_{O L D C}}{\frac{f_{z 1}}{f_{p n d 1}}} f_{p d}=\frac{A_{O L D C}}{f_{z 1}} f_{p d} f_{p n d 1} .
$$

2) Case (b), Dominant $C_{L}$ : In this case, $f_{z 1}$ is smaller than $f_{p n d 1}$ [Fig. 4(b)]. Note that $f_{z 1}$ and $f_{p d}$ change at the same rate, while $f_{\text {pnd } 1}$ remains approximately constant with changes in $C_{L}$. The response in blue broken lines shows the equivalent one pole response obtained by increasing the open-loop gain by a factor $\triangle A_{O L}$ from $A_{O L D C}$ to a value $A_{O L D C} C^{\prime}$ where $\triangle A_{O L}$ is given by

$$
\begin{aligned}
\Delta A_{O L} & =\Delta f=\frac{f_{z 1}}{f_{p n d 1}} \\
A_{O L D C^{\prime}} & =\frac{A_{O L D C}}{\Delta A_{O L}}=\frac{A_{O L D C}}{\frac{f_{z 1}}{f_{p n d 1}}} .
\end{aligned}
$$

The equivalent dominant pole of the response in blue broken lines is again $f_{p d}$ which leads to a unity-gain frequency $f_{U G}$ given by

$$
f_{U G}=A_{O L D C^{\prime}} f_{p d}=\frac{A_{O L D C}}{\frac{f_{z 1}}{f_{p n d 1}}} f_{p d}=\frac{A_{O L D C}}{f_{z 1}} f_{p d} f_{p n d 1} .
$$



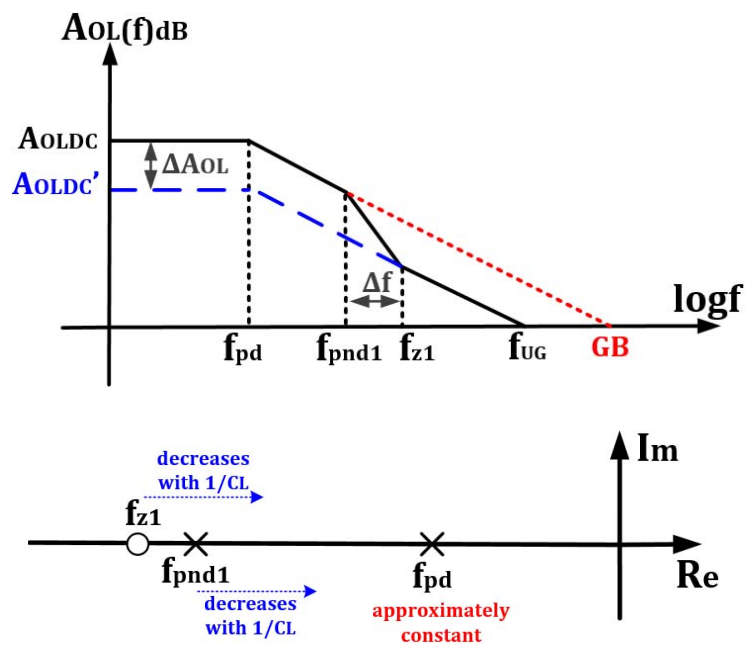

(a)
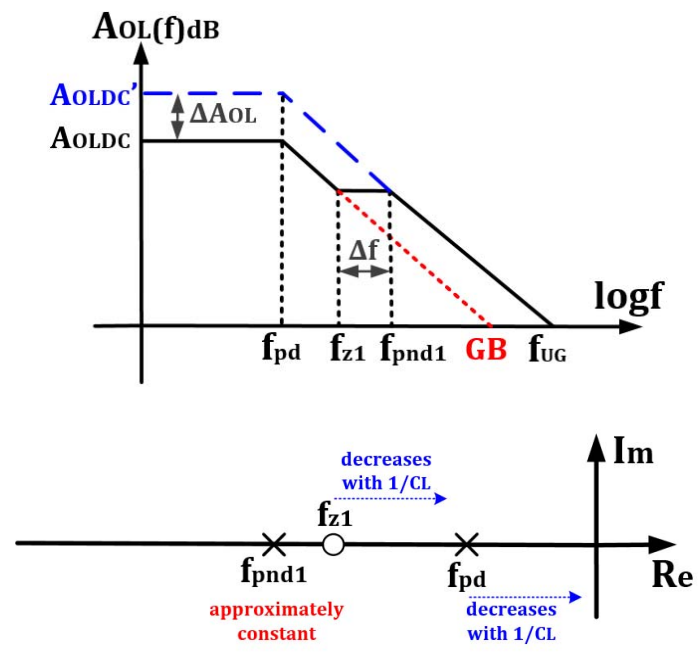

(b)

Fig. 4. Open-loop response (top) and pole-zero location (bottom) of the proposed circuit. (a) $f_{z 1}$ larger than $f_{p n d 1}$. (b) $f_{z 1}$ smaller than $f_{p n d 1}$.

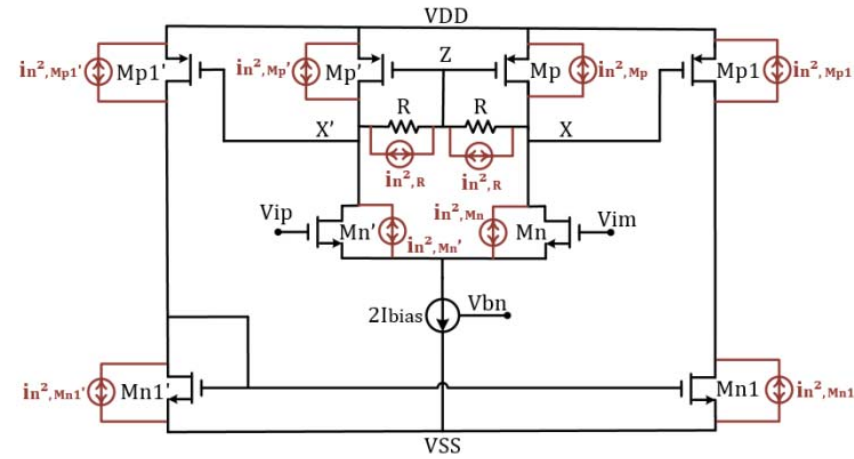

Fig. 5. Simplified model for input-referred noise of the proposed class-AB-AB op-amp of Fig. 2 including dominant noise sources.

Using the general expressions for $A_{O L D C}, f_{z 1}, f_{p d}$, and $f_{p n d 1}$ from Table I, (10) and (13) are reduced to

$$
f_{U G}=\frac{g_{m e f f} g_{m I I} R_{S}}{2 \pi C_{c}\left(1+g_{m I I} R_{S}\right)} .
$$

It can be seen that in spite of the fact that $f_{z 1}, f_{p n d 1}$, and $f_{p d}$ are all dependent on $C_{L}$, the unity-gain frequency $f_{U G}$ which approximately corresponds to the closed-loop $B W$ at $V_{\text {out }}$ remains the same and independent on $C_{L}$.

\section{Stability}

The $P M$ of the proposed circuit can be expressed by

$$
P M \approx 90^{\circ}-\tan ^{-1}\left(\frac{f_{U G}}{f_{p n d 1}}\right)+\tan ^{-1}\left(\frac{f_{U G}}{f_{z 1}}\right) .
$$

Consider the following cases.

1) Dominant $C_{L}$ [Fig. 4(b)]: In this case, stability is enforced since $f_{z 1}<f_{p n d 1}$ and the phase shift of $f_{z 1}$ mostly cancels the phase shift of the dominant pole $f_{p d}$, the only remaining phase shift at the unity-gain frequency is due to $f_{p n d 1}$. This leads always to a high $P M$ for large $C_{L}$ values.

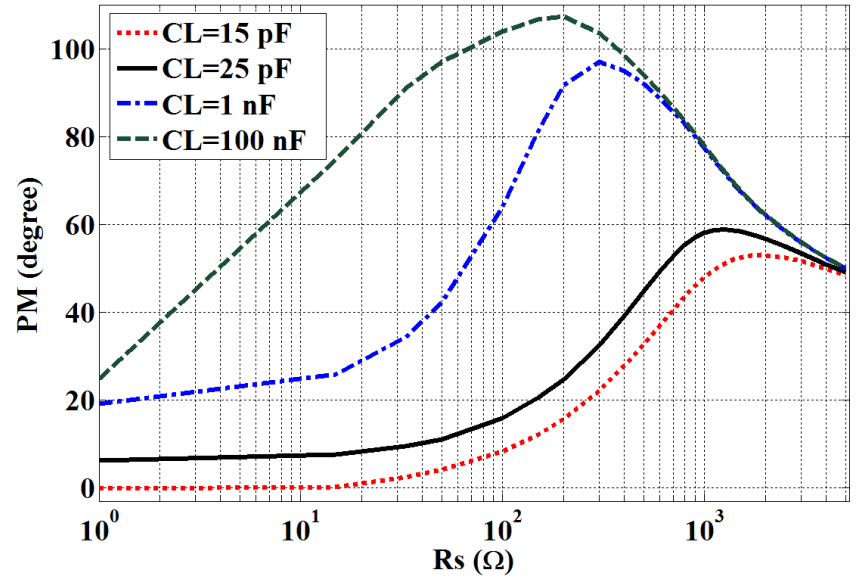

Fig. 6. Simulated $P M$ of the proposed circuit with $R_{S}$ changing over the range $1 \Omega<R_{S}<5 \mathrm{k} \Omega$ and four different values $C_{L}=15 \mathrm{pF}, 25 \mathrm{pF}, 1 \mathrm{nF}$, and $100 \mathrm{nF}$.

TABLE II

\begin{tabular}{|c|c|c|c|}
\hline Parameter & Value & Parameter & Value \\
\hline$(\mathrm{W} / \mathrm{L})_{\mathrm{NMOS}}(\mu \mathrm{m} / \mu \mathrm{m})$ & $20 / 0.7$ & $\mathrm{C}_{\mathrm{c}}(\mathrm{pF})$ & 10 \\
\hline$(\mathrm{W} / \mathrm{L})_{\mathrm{PMOS}}(\mu \mathrm{m} / \mu \mathrm{m})$ & $100 / 0.7$ & $\mathrm{C}_{\mathrm{X}}(\mathrm{pF})$ & 0.4 \\
\hline $\mathbf{g}_{\mathrm{mDP}}(\mu \mathrm{A} / \mathbf{V})$ & 109 & $R_{z}(k \Omega)$ & 6 \\
\hline $\mathrm{g}_{\mathrm{mp} 1}(\mu \mathrm{A} / \mathrm{V})$ & 137 & $R_{0 I}(k \Omega)$ & 435 \\
\hline $\mathrm{g}_{\mathrm{mpo}}(\boldsymbol{\mu} \mathbf{A} / \mathbf{V})$ & 428 & $\mathrm{~g}_{\mathrm{mII}}(\mu \mathbf{A} / \mathbf{V})^{*}$ & 866 \\
\hline $\mathrm{g}_{\mathrm{mno}}(\mu \mathrm{A} / \mathrm{V})$ & 438 & $R_{\text {oII }}(k \Omega)$ & 125 \\
\hline $\mathrm{g}_{\mathrm{meff}}(\mu \mathrm{A} / \mathrm{V})$ & 1724 & $\mathbf{g}_{\text {mpinv }}(\mu \mathrm{A} / \mathrm{V})$ & 73.8 \\
\hline $\mathrm{R}(\mathrm{k} \Omega)$ & 150 & $g_{\operatorname{mninv}}(\mu \mathrm{A} / \mathrm{V})$ & 74.3 \\
\hline
\end{tabular}

Parameter Values of the Proposed Circuit [Fig. 2(b)]

* at low frequencies $g_{m I I}=g_{m p o}$ and at high frequencies $g_{m I I}=g_{m p o}+g_{m n o}$.

2) Dominant $C_{c}$ [Fig. 4(a)]: In this case, $f_{z 1}>f_{p n d 1}$, and the ratio $f_{z 1} / f_{\text {pnd } 1}=\left(1+g_{m I I} R_{S}\right) / g_{m I I} R_{S}$ is constant independent of $C_{L}$. However, they both shift closer to the unity-gain frequency $f_{U G}$ as $C_{L}$ decreases 
This is the author's version of an article that has been published in this journal. Changes were made to this version by the publisher prior to publication. The final version of record is available at https://doi.org/10.1109/TVLSI.2018.2830365

TABLE III

Simulated Pole and Zeroes in the Proposed Circuit of Fig. 2(b) For Three Different Capacitive Loads

\begin{tabular}{|c|c|c|c|c|}
\hline Pole-Zeroes & $\begin{array}{c}\text { Dominant } C_{c}: \\
C_{L}=25 \mathrm{pF}\end{array}$ & $\begin{array}{c}\text { Neither } C_{c} \text { nor } C_{L} \\
\text { dominant: } \\
C_{L}=1 \mathrm{nF}\end{array}$ & $\begin{array}{c}\text { Dominant } C_{L} \text { : } \\
C_{L}=10 \mathrm{nF}\end{array}$ & $\begin{array}{c}\text { Changing with } \\
C_{L} ? ?\end{array}$ \\
\hline$f_{p d}$ & $733 \mathrm{~Hz}$ & $465 \mathrm{~Hz}$ & $105 \mathrm{~Hz}$ & Yes \\
\hline$f_{\text {pndl }}$ & $1.94 \mathrm{MHz}$ & $73.2 \mathrm{kHz}$ & $32.4 \mathrm{kHz}$ & Yes \\
\hline$f_{\text {pnd2 }}$ & $3.62 \mathrm{MHz}$ & $3.62 \mathrm{MHz}$ & $3.62 \mathrm{MHz}$ & No \\
\hline$f_{z 1}$ & $7.07 \mathrm{MHz}$ & $176.8 \mathrm{kHz}$ & $17.7 \mathrm{kHz}$ & Yes \\
\hline$f_{z 2}$ & $3.05 \mathrm{MHz}$ & $3.05 \mathrm{MHz}$ & $3.05 \mathrm{MHz}$ & No \\
\hline$f_{U G}$ & $12.05 \mathrm{MHz}$ & $12.05 \mathrm{MHz}$ & $12.05 \mathrm{MHz}$ & No \\
\hline
\end{tabular}

TABLE IV

Simulated Settling Time of the Proposed Op-Amp of Fig. 2(b) By Adding $\pm 20 \%$ Mismatches TO THE NOMinal VALUES OF $C_{c}, R_{z}, C_{X}$, AND $R$

\begin{tabular}{|c|c|c|c|c|c|}
\hline $\begin{array}{c}\text { Cases: } \\
\mathbf{- 2 0} \%<\text { mismatches }<+\mathbf{2 0} \%\end{array}$ & $\boldsymbol{C}_{\boldsymbol{c}} \mathbf{( p F )}$ & $\boldsymbol{R}_{\boldsymbol{z}}(\mathbf{k} \boldsymbol{\Omega})$ & $\boldsymbol{C}_{\boldsymbol{X}}(\mathbf{p F})$ & $\boldsymbol{R} \mathbf{( k \boldsymbol { \Omega } )}$ & $\begin{array}{c}\text { Settling time (ns) } \\
\mathbf{( 0 . 5 \% )}\end{array}$ \\
\hline a: (nominal values) & 10 & 6 & $\approx 0.4$ & 150 & 156 \\
\hline $\mathbf{b :}$ & 8 & 4.8 & $\approx 0.48$ & 180 & 148 \\
\hline c: & 12 & 7.2 & $\approx 0.48$ & 120 & 175 \\
\hline d: & 10 & 4.8 & $\approx 0.4$ & 120 & 155 \\
\hline e: & 12 & 6 & $\approx 0.48$ & 180 & 166 \\
\hline f: & 8 & 7.2 & $\approx 0.4$ & 150 & 149 \\
\hline \hline
\end{tabular}

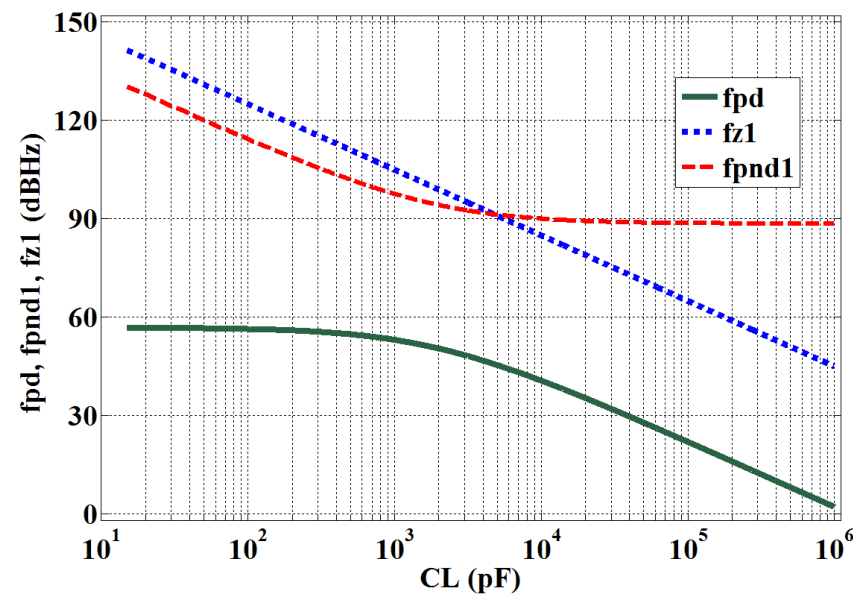

Fig. 7. Locations of dominant pole $f_{p d}$, nondominant pole $f_{p n d 1}$, and zero $f_{z 1}$ for $R_{S}=900 \Omega$ over the $C_{L}$ range $15 \mathrm{pF}<C_{L}<1 \mu \mathrm{F}$.

which decreases $P M$ and consequently the lowest $P M$ corresponds to $C_{\operatorname{Lmin}}$. For a given value $C_{\text {Lmin }}$ it is possible to find a value $R_{S}$ that satisfies stability with a reasonable $P M$ as it is illustrated with a design example in Section III-A. The phase-lead compensation resistor $R_{S}$ determines also the maximum output current which has a value $I_{\text {out MAX }}=V_{\text {out MAX }} / R_{S} \approx V_{D D} / R_{S}$ and this limits the maximum value of $R_{S}$.

In practice, other high frequency poles neglected in the analysis slightly degrade $P M$. Note that as opposed to conventional Miller op-amps, the combination of Miller and phaselead compensation allows to design pseudo two-stage op-amps with high $P M$ over an extremely wide range capacitive loads starting from low $C_{L}$ values.

\section{Slew Rate Analysis}

Based on the discussion made in Section II-A, the maximum voltage variation at nodes $X$, and $X^{\prime}\left(\triangle V_{X, M A X}\right)$, the maximum source-gate voltage variations of transistors $\operatorname{Mp} 1\left(\Delta V_{V S G M p 1 M A X}\right)$ and Mpinv $\left(\Delta V_{V S G M p i n v M A X}\right)$ and also the maximum currents at nodes $V_{I}\left(I_{\text {out }} V_{I}, M A X\right)$ and $V_{I}^{\prime}\left(I_{\text {out } V_{I}}, M_{A X}\right)$ are defined as follows:

$$
\begin{aligned}
\Delta V_{X, M A X} & =\Delta V_{V S G M p 1 M A X} \\
& =\Delta V_{V S G M \text { pinv } M A X}=I_{\text {bias }} R \\
I_{\text {out } V_{I}, M A X} & =K_{M p 1}\left(V_{S G M p 1}^{Q}-\left|V_{\text {thP } P}\right|+\Delta V_{V S G M p 1 M A X}\right)^{2} \\
& =K_{M p 1}\left(V_{S D \text { sat Mp } 1}+I_{\text {bias }} R\right)^{2} \\
I_{\text {out } V_{I}{ }^{\prime}, M A X} & =K_{M p i n v}\left(V_{\text {SGMpinv }}^{Q}-\left|V_{\text {thP }}\right|+\Delta V_{V S G M p i n v M A X}\right)^{2} \\
& =K_{\text {Mp } 1}\left(V_{\text {SDsatMpinv }}+I_{\text {bias }} R\right)^{2}
\end{aligned}
$$

where

$$
K_{p}=\frac{\mu_{p} C_{o x}}{2} \frac{W}{L} .
$$

Further, $V_{t h P}$ and $\mu_{p}$ are the nominal value of the threshold voltage and the mobility for pMOS, respectively. Also $C_{o x}$ is the gate capacitance of the MOSFET. Maximum currents at nodes $V_{I}$, and $V_{I}^{\prime} ; I_{\text {out } V I, M A X}$, and $I_{\text {out } V I^{\prime}, M A X} \gg 2 I_{\text {bias }}$ lead to large source-gate (gate-source) variations in pMOS and nMOS output transistors $\left(\triangle V_{I_{M A X}}=\Delta V_{V S G \text { poMAX }} \approx V_{S S}\right.$ and $\left.\triangle V_{I^{\prime} M A X}=\Delta V_{V G S n o M A X} \approx V_{D D}\right)$.

These large voltage variations at nodes $V_{I}$ and $V_{I}^{\prime}$ result in large values of $I_{\text {out MAX }}^{+}$and $I_{\text {out MAX }}^{-}$. However, the maximum output current $I_{\text {outMAX }}=\min \left(I_{\text {outMAX }}^{+}\right.$and $\left.I_{\text {out MAX }}^{-}\right)$is 


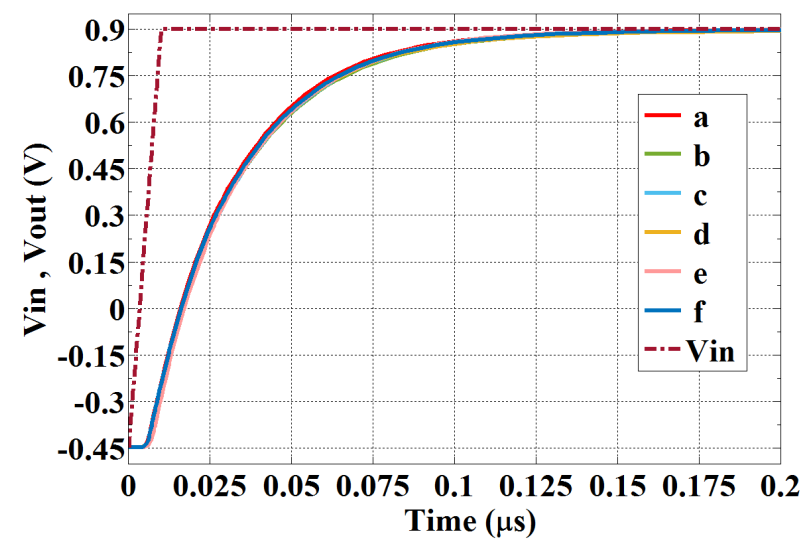

Fig. 8. Simulated settling time of the proposed class-AB-AB op-amp of Fig. 2(b) for all cases of mismatches shown in Table IV. The input signal is a $1.35-\mathrm{V}_{\mathrm{p}-\mathrm{p}}$ square waveform, for $C_{L}=25 \mathrm{pF}$.

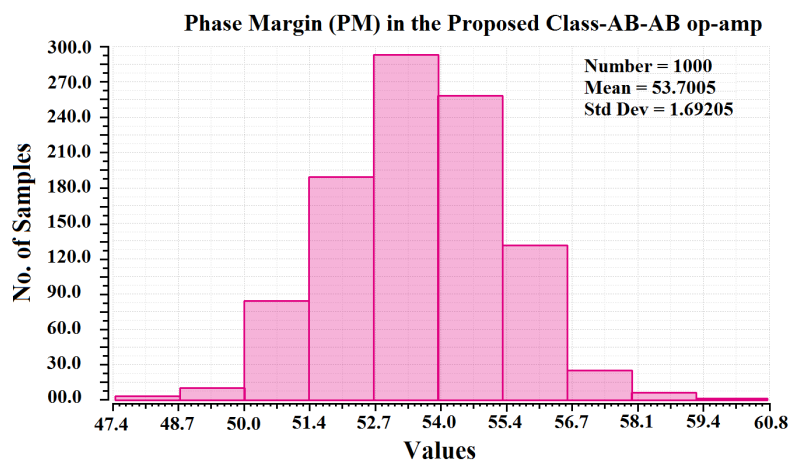

Fig. 9. Checking robustness of $P M$ by performing 1000 Monte Carlo runs including the process and mismatch data $\left(C_{L}=25 \mathrm{pF}\right)$.

limited by the phase-lead compensation resistor $R_{S}$

$$
I_{\text {out } M A X}=\frac{V_{\text {out } M A X}}{R_{S}} \approx \frac{V_{D D}}{R_{S}} .
$$

Furthermore, the $S R$ of the proposed class-AB-AB op-amp can be defined in the following equation:

$$
S R \approx \frac{I_{\text {out } M A X}}{C_{c}+C_{L}}=\frac{V_{D D}}{R_{S}\left(C_{c}+C_{L}\right)}
$$

\section{E. Noise Analysis}

Considering that noise is provided mainly by the composite first stage, the model including noise sources of the proposed op-amp of Fig. 2 is shown in Fig. 5.

Current noise $i_{n, M i}^{2}$ in each MOSFET can be modeled by thermal noise $\left.\overline{\left(i_{n, \text { th }}^{2}\right.}=4 k T \gamma g_{m M i}\right)$ and flicker noise $\overline{\left(i_{n, 1 / f}^{2}\right.}=$ $\left(K\left(g_{m M i}\right)^{2}\right) /\left(\left(C_{o x} W L f\right)\right)$ and in each resistor $R$ can be modeled by thermal noise $\left.\overline{\left(i_{n, R}^{2}\right.}=4 k T / R\right)$

$$
\overline{i_{n, \mathrm{Mi}}^{2}}=\overline{i_{n, \mathrm{th}}^{2}}+\overline{i_{n, \frac{1}{f}}^{2}} \text {. }
$$

Further $k, T, \gamma$, and $g_{m M i}$ are Boltzmann's constant $(k=$ $\left.1.38 \times 10^{-23} \mathrm{~J} / \mathrm{K}\right)$, the absolute temperature $(T=300 \mathrm{~K})$, transconductance gain of transistor, and channel thermal noise coefficient $(\gamma \approx 2 / 3)$, respectively. In addition, $K, C_{o x}, W, L$, and $f$ are the process-dependent constant $\left(K=10^{-25} \mathrm{~V}^{2} \cdot \mathrm{F}\right)$,

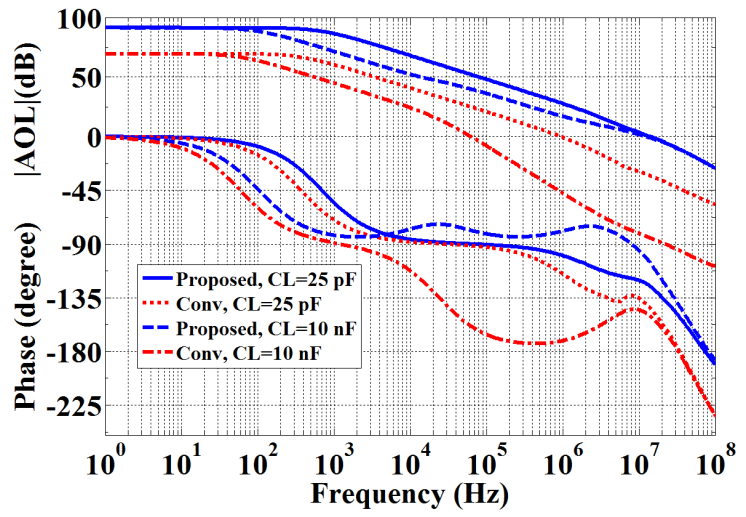

(a)

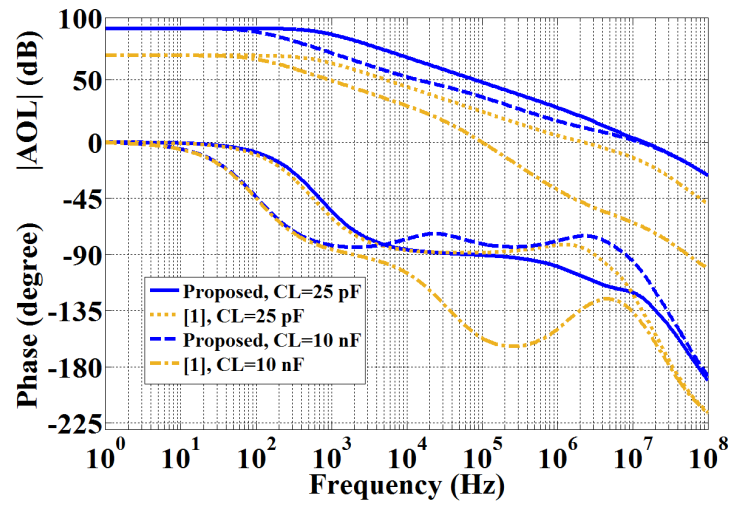

(b)

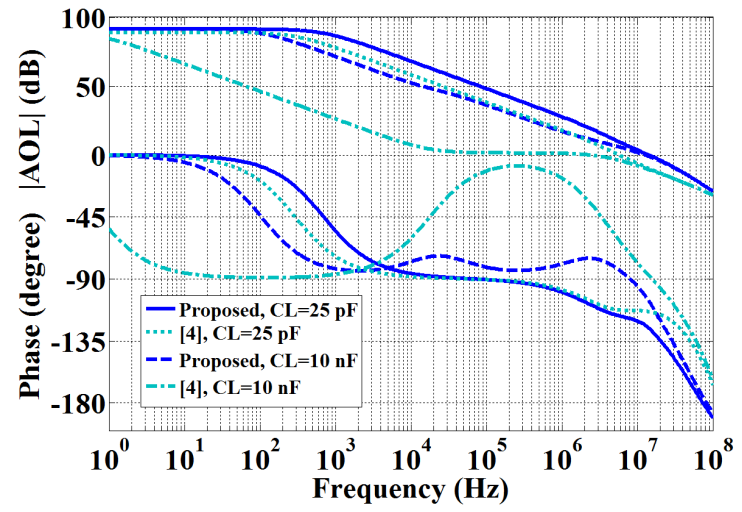

(c)

Fig. 10. Simulated open-loop response for two capacitive loads $C_{L}=25 \mathrm{pF}$ and a large-size $C_{L}=10 \mathrm{nF}$. (a) Response of the proposed and conventional op-amps. (b) Response of the proposed op-amp and scheme in [1]. (c) Response of the proposed op-amp and the circuit in [4].

oxide capacitance, width of transistor, length of transistor's channel and frequency, respectively.

The equivalent input-referred noise is derived from the following equation:

$$
\begin{aligned}
& \overline{v_{n, \mathrm{in}}^{2}} \\
& =\frac{1}{\left(g_{m n, n^{\prime}}\right)^{2}}\left[\overline{i_{n, \mathrm{Mn}}^{2}}+\overline{i_{n, \mathrm{Mn}^{\prime}}^{2}}+\overline{i_{n, \mathrm{Mp}}^{2}}+\overline{i_{n, \mathrm{Mp}^{\prime}}^{2}}+\overline{i_{n, R}^{2}}+\overline{i_{n, R}^{2}}\right. \\
& \left.\quad+\frac{1}{\left(g_{m p 1, p 1^{\prime}} R\right)^{2}}\left(\overline{i_{n, \mathrm{Mn} 1}^{2}}+\overline{i_{n, \mathrm{Mn} 1^{\prime}}^{2}}+\overline{i_{n, \mathrm{Mp} 1}^{2}}+\overline{i_{n, \mathrm{Mp}^{\prime}}^{2}}\right)\right] .
\end{aligned}
$$


TABLE V

Corner Simulation Results of the Proposed Circuit [FIG. 2(b)] $\left(C_{L}=25 \mathrm{pF}, R_{S}=900 \Omega, V_{D D}=0.9 \mathrm{~V}\right.$, $V_{S S}=-0.9 \mathrm{~V}$, AND $\left.I_{\text {bias }}=5 \mu \mathrm{A}\right)$

\begin{tabular}{|c|c|c|c|c|c|}
\hline $\begin{array}{l}\text { Corner: } \\
\mathbf{T}=27^{\circ} \mathrm{C}\end{array}$ & TT & SS & FF & SF & FS \\
\hline $\mathbf{I}_{\text {totbias }}(\boldsymbol{\mu A})$ & 42.67 & 40.4 & 46.06 & 42.24 & 43.57 \\
\hline $\mathbf{f}_{\mathrm{UG}}(\mathrm{MHz})$ & 12.05 & 11.25 & 13.38 & 12.19 & 12.16 \\
\hline $\mathbf{I}_{\text {outMAX }}(\boldsymbol{\mu A})$ & 995 & 955 & 1088 & 942 & 1085 \\
\hline$\frac{\text { FOM }_{\mathbf{G}}}{(\sqrt{\mu \mathrm{AMHzpF}} / \mu \mathrm{W})}$ & 7.13 & 7.13 & 7.28 & 7.05 & 7.32 \\
\hline $\mathbf{A}_{\text {OLDC }}(\mathbf{d B})$ & 91.2 & 91 & 91.27 & 91.2 & 91.1 \\
\hline $\mathbf{P M}\left({ }^{\circ}\right)$ & 58.4 & 58 & 58.9 & 58.6 & 58.5 \\
\hline THD ${ }^{*}(\%)$ & 0.094 & 0.123 & 0.086 & 0.110 & 0.090 \\
\hline $\begin{array}{l}\text { Corner: } \\
\mathrm{T}=125^{\circ} \mathrm{C}\end{array}$ & TT & SS & FF & SF & FS \\
\hline $\mathbf{I}_{\text {totbias }}(\boldsymbol{\mu A})$ & 39.84 & 37.12 & 43.5 & 38.6 & 41.37 \\
\hline $\mathbf{f}_{\mathrm{UG}}(\mathrm{MHz})$ & 10.84 & 10.30 & 11.32 & 10.58 & 8.87 \\
\hline $\mathbf{I}_{\text {outMAXX }}(\mu A)$ & 933 & 882 & 961 & 877 & 1004 \\
\hline$\frac{\text { FOM }_{\mathbf{G}}}{(\sqrt{\mu \mathrm{AMHzpF}} / \boldsymbol{\mu W})}$ & 7.01 & 7.13 & 6.66 & 6.93 & 6.34 \\
\hline $\mathbf{A}_{\text {OLDC }}(\mathrm{dB})$ & 90.6 & 90.4 & 90.6 & 90.4 & 90.6 \\
\hline $\mathbf{P M}\left(^{\circ}\right)$ & 58.6 & 57.4 & 55 & 59.2 & 58 \\
\hline THD $^{*}(\%)$ & 0.106 & 0.116 & 0.097 & 0.111 & 0.101 \\
\hline $\begin{array}{l}\text { Corner: } \\
\mathrm{T}=-40^{\circ} \mathrm{C}\end{array}$ & TT & SS & FF & SF & FS \\
\hline $\mathbf{I}_{\text {totbias }}(\boldsymbol{\mu A})$ & 48.9 & 50.43 & 52.21 & 52.39 & 49.1 \\
\hline $\mathbf{f}_{\mathrm{UG}}(\mathrm{MHz})$ & 10.57 & 10.44 & 10.28 & 10.10 & 10.73 \\
\hline$I_{\text {outMAX }}(\mu \mathrm{A})$ & 1036 & 932 & 1094 & 972 & 1088 \\
\hline$\frac{\text { FOM }_{\mathbf{G}}}{(\sqrt{\mu \mathbf{A M H z p F}} / \boldsymbol{\mu W})}$ & 5.94 & 5.43 & 5.64 & 5.25 & 6.11 \\
\hline $\mathbf{A}_{\text {OLDC }}(\mathrm{dB})$ & 91.3 & 91 & 91.4 & 91.2 & 91.2 \\
\hline PM $\left(^{\circ}\right)$ & 51.7 & 45.5 & 51.1 & 46.2 & 52.8 \\
\hline THD* $(\%)$ & 0.121 & 0.4 & 0.081 & 0.278 & 0.087 \\
\hline
\end{tabular}

Total harmonic distortion THD-@100 kHz, 1-Vp-p input signal.

Note that in (23) the effect of shell transistors' noise can be neglected since it is reduced by a factor $\left(g_{m p 1, p 1^{\prime}} R\right)^{2}$. Considering this and also assuming $g_{m n, n^{\prime}}=g_{m D P}$, simplifies (23) to (24)

$$
\begin{aligned}
\overline{v_{n, i n}^{2}}= & \frac{2\left[\overline{i_{n, \mathrm{Mn}}^{2}}+\overline{i_{n, \mathrm{Mp}}^{2}}+\overline{i_{n, R}^{2}}\right]}{\left(g_{m D P}\right)^{2}} \\
= & 2 \frac{4 k T \gamma}{g_{m D P}}+\frac{K}{\left(C_{o x} W_{n} L f\right)}+\frac{4 k T \gamma g_{m p}}{\left(g_{m D P}\right)^{2}} \\
& \left.+\frac{K\left(g_{m p}\right)^{2}}{\left(\left(C_{o x} W_{p} L f\right)\left(g_{m D P}\right)^{2}\right)}+\frac{4 k T}{R\left(g_{m D P}\right)^{2}}\right] .
\end{aligned}
$$

It can be seen that differential pair transistors $\left(\mathrm{Mn}, \mathrm{Mn}^{\prime}\right)$ have dominant effect on the equivalent input-referred noise of the proposed op-amp.

\section{Simulation And Measurement Results}

\section{A. Simulation Results}

The proposed circuit of Fig. 2(b) was designed in a 180-nm CMOS technology and simulated with dual supplies

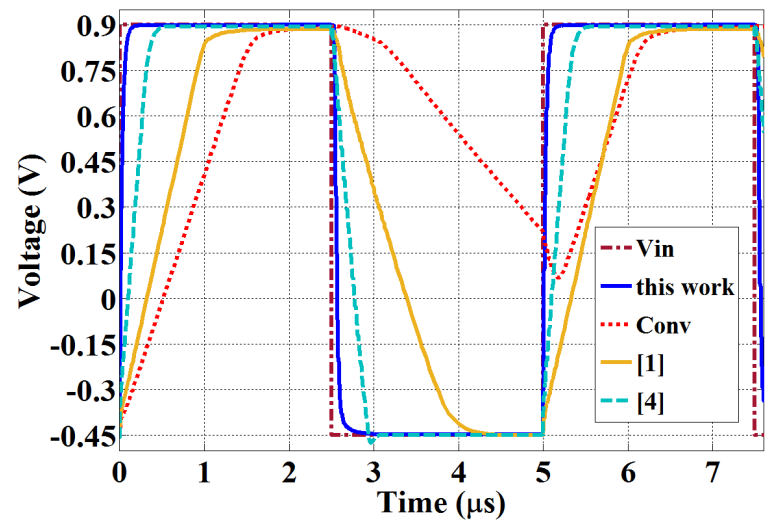

(a)

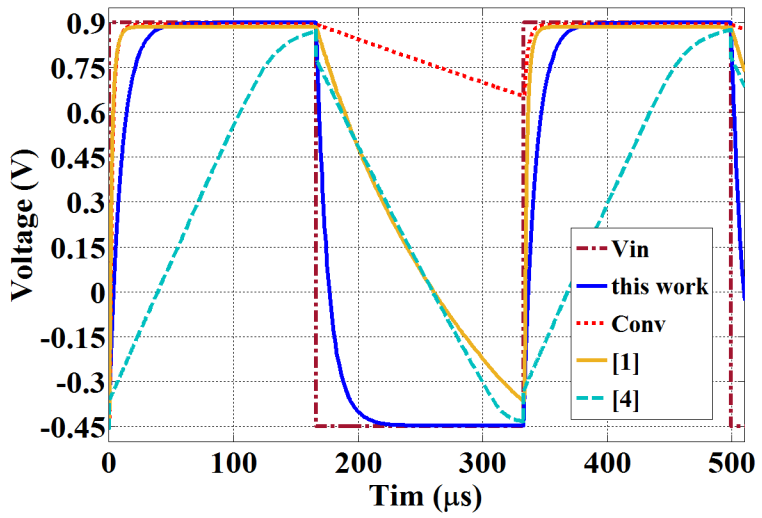

(b)

Fig. 11. Simulated transient response of the proposed class-AB-AB op-amp of Fig. 2(b) compared to the conventional Miller op-amp and also amplifiers in [1] and [4]. The input is a $1.35-\mathrm{V}_{\mathrm{p}-\mathrm{p}}$ square waveform. (a) For $C_{L}=25 \mathrm{pF}$. (b) For a large size, $C_{L}=10 \mathrm{nF}$.

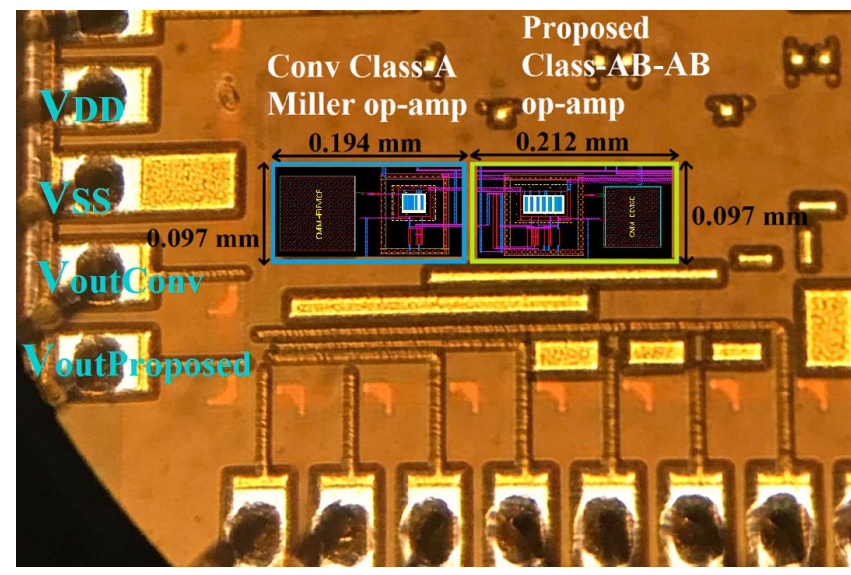

Fig. 12. Micrograph of fabricated chip in 180-nm CMOS technology. It includes the proposed class-ABs-AB Gm-enhanced op-amp [Fig. 2(b)] and a conventional two-stage Miller op-amp (Fig. 1).

$V_{D D}=+0.9 \mathrm{~V}$ and $V_{S S}=-0.9 \mathrm{~V}$, threshold voltages $V_{t h P} \approx V_{t h N} \approx 0.45 \mathrm{~V}$, and $I_{\text {bias }}=5 \mu \mathrm{A}$. Transistors' dimensions, output resistances, and small-signal transconductance gains $\left(R_{O I}, R_{O I I}\right.$, and $\left.g_{m}\right)$ are listed in Table II. The parasitic capacitance at node $X\left(C_{X}\right)$ is calculated from

$C_{X} \approx C_{g s M p 1}+C_{g s M p i n v}+C_{g d M p}+C_{g d M n}+C_{d b M n}+C_{d b M p}$. 

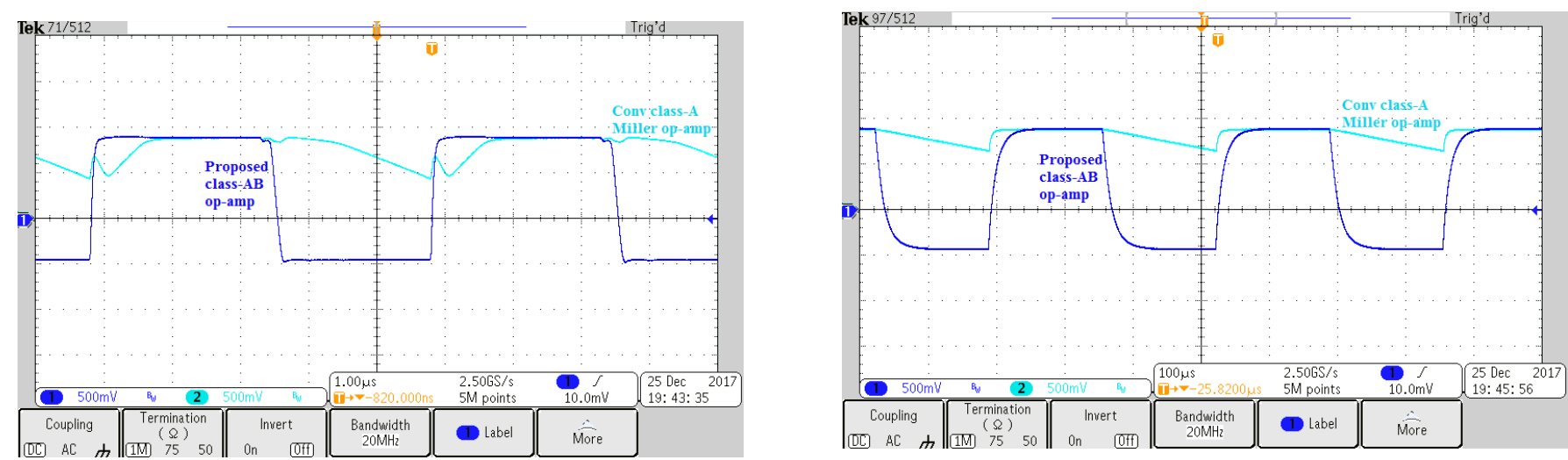

(a)
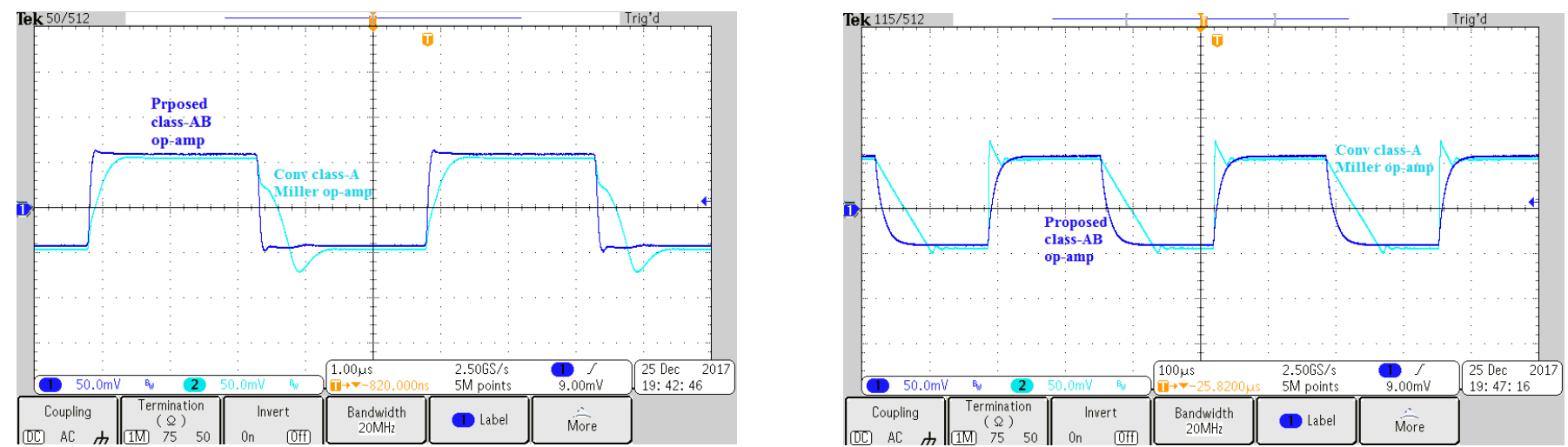

(b)

Fig. 13. Experimental transient response of the proposed class-AB-AB and conventional class-A Miller op-amps to two different input square waveforms. (a) $1.35-\mathrm{V}_{\mathrm{p}-\mathrm{p}}$ input square waveform, for $C_{L}=25 \mathrm{pF}$ (left) and for $C_{L}=10 \mathrm{nF}$ (right). (b) $0.1-\mathrm{V}_{\mathrm{p}-\mathrm{p}}$ input square waveform, for $C_{L}=25 \mathrm{pF}$ (left) and for $C_{L}=10 \mathrm{nF}$ (right).

With $C_{o x}=8.78 \mathrm{fF} / \mu \mathrm{m}^{2}$ in $0.18-\mu \mathrm{m} \mathrm{CMOS}$ and transistors' dimensions in Table II result in a value $C_{X} \approx 0.4$ pF. By selecting $C_{c}=10 \mathrm{pF}$, a value $R_{z}=6 \mathrm{k} \Omega$ is obtained from $R_{z}=\left(R C_{X} / C_{c}\right)+\left(1 / g_{m I I}\right)$ in order to achieve $C_{L}$-independent pole-zero cancellation of $f_{\text {pnd } 2} \approx f_{z 2} \sim$ (3-4) $\mathrm{MHz}$ (see Section II-B).

The next step is to determine the value of the phaselead resistance $R_{S}$ which is critical for stability and also for the maximum output current $\left(I_{\text {out } M A X}=V_{\text {out } M A X} / R_{S}\right)$. Fig. 6 shows the simulated $P M$ in the proposed circuit with $R_{S}$ changing from $1 \Omega$ to $5 \mathrm{k} \Omega$ and $C_{L}$ parametrized with values $C_{L}=15 \mathrm{pF}, 25 \mathrm{pF}, 1 \mathrm{nF}$, and $100 \mathrm{nF}$. It can be seen in Fig. 6 that as expected, in the range shown for $R_{S}$, higher $C_{L}$ values lead to higher $P M$. For $R_{s} \geq 800 \Omega$, a $P M>45^{\circ}$ results over the entire range of $C_{L}$ values. In this paper, $R_{S}=$ $900 \Omega$ was selected, which leads to $I_{\text {out } M A X} \approx V_{D D} / R_{S}=1$ $\mathrm{mA}$ and a $P M>45^{\circ}$ for $C_{L}>15 \mathrm{pF}$. For lower maximum output currents $I_{\text {outMAX }}$ a higher resistance $R_{S}$ can be used. This allows utilization of lower $C_{\text {Lmin }}$ values.

Fig. 7 shows the dominant pole $f_{p d}$, nondominant pole $f_{p n d 1}$, and zero $f_{z 1}$ for $R_{s}=900 \Omega$ over the range of $15 \mathrm{pF}<C_{L}<1 \mu \mathrm{F}$. It can be seen that the change in their location is in agreement with the discussion in Sections II-B and II-C.

Table III provides the simulated values of poles, zeroes, and $f_{U G}$ for three different capacitive loads: $C_{L}=25 \mathrm{pF}$
$\left(C_{L} \ll g_{m I I} R_{O I} C_{c}\right.$ : dominant $\left.C_{c}\right), C_{L}=1 \mathrm{nF}\left(C_{L} \approx\right.$ $\left.g_{m I I} R_{O I} C_{c}\right)$, and $C_{L}=10 \mathrm{nF}\left(C_{L} \gg g_{m I I} R_{O I} C_{c}\right.$ : dominant $C_{L}$ ). Note that the simulated values of the pole-zeroes closely follow their expressions listed in Table I. As expected, in all simulations with different capacitive loads $C_{L}$, the values of $f_{z 2}$ and $f_{p n d 2}$ remain constant and close to each other; $f_{z 2}=3.05 \mathrm{MHz}$ and $f_{p n d 2}=3.62 \mathrm{MHz}$. This validates the discussion made about $C_{L}$-independent pole-zero cancelation in Section II-B.

Pole-zero cancellation can lead to long settling time when there is large mismatch between the pole and zero [27]. In the proposed circuit, the mismatch between $C_{L}$-independent $f_{z 2}$ and $f_{p n d 2}$ is determined by manufacturing tolerances of the order of $20 \%$ for each element (i.e., $C_{c}, R, C_{X}, R_{z}, \ldots$ ). This mismatch as well as the location of $f_{z 2}$ and $f_{p n d 2}$ relative to the location of $f_{U G}$ does not have significant effect on the settling time. This is verified with transient simulations shown in Fig. 8 and Table IV, where $C_{L}=25 \mathrm{pF}$ and $f_{z 2}$ and $f_{p n d} 2$ are purposely mismatched by adding capacitances connected to nodes $X$ and $X^{\prime}$, and also manipulating the values of $C_{c}, R_{z}$, and $R$ by $\pm 20 \%$. It can be seen that in all cases (a, b, c, d, e, and f) a negligible effect on transient response is observed. The input signal is a $1.35-\mathrm{V}_{\mathrm{p}-\mathrm{p}}$ square waveform. Above discussion applies to a wide range of $C_{L}$ loads since the values of $f_{z 2}$ and $f_{p n d 2}$ are not dependent on $C_{L}$. 
This is the author's version of an article that has been published in this journal. Changes were made to this version by the publisher prior to publication. The final version of record is available at https://doi.org/10.1109/TVLSI.2018.2830365

TABLE VI

Measurement Results of the Proposed Class-AB-AB Op-Amp And Comparison to Other References

\begin{tabular}{|c|c|c|c|c|c|c|c|c|c|c|c|c|c|}
\hline Parameters & $\begin{array}{l}\text { Conv. } \\
\text { Miller } \\
\text { op-amp }\end{array}$ & $\begin{array}{c}{[4]} \\
(2002)\end{array}$ & $\begin{array}{c}{[6]} \\
(2005)\end{array}$ & $\begin{array}{c}{[2]} \\
(2011)\end{array}$ & $\begin{array}{c}{[5]} \\
(2012)\end{array}$ & $\begin{array}{c}{[1]} \\
(2014)\end{array}$ & $\begin{array}{c}{[7]} \\
(2016)\end{array}$ & $\begin{array}{c}{[3]} \\
(2016)\end{array}$ & $\begin{array}{c}{[8]} \\
(2017)\end{array}$ & $\begin{array}{c}{[9]} \\
\text { (2017) }\end{array}$ & $\begin{array}{c}{[10]} \\
(2017)\end{array}$ & \multicolumn{2}{|c|}{$\begin{array}{c}\text { This } \\
\text { work } \\
\text { Fig. 2(b) }\end{array}$} \\
\hline CMOS Process $(\mu \mathrm{m})$ & 0.18 & 0.5 & 0.5 & 0.13 & 0.5 & 0.35 & 0.18 & 0.18 & 0.5 & 0.18 & 0.18 & \multicolumn{2}{|c|}{0.18} \\
\hline Single-Stage & No & Yes & Yes & No & No & No & Yes & No & Yes & Yes & Yes & \multicolumn{2}{|c|}{ No } \\
\hline Supply (V) & \pm 0.9 & \pm 2.5 & \pm 1 & 1.2 & \pm 1.25 & \pm 1.65 & 1.8 & 0.7 & \pm 1 & \pm 0.9 & \pm 0.9 & \multicolumn{2}{|c|}{ \pm 0.9} \\
\hline $\mathbf{I}_{\text {totbias }}(\boldsymbol{\mu} \mathbf{A})$ & 20.78 & 160 & 60 & 91.67 & 175 & 420 & 6611.1 & 36.28 & 60 & 8 & 40 & \multicolumn{2}{|c|}{43} \\
\hline $\mathbf{P}_{\text {diss }}(\mu \mathrm{W})$ & 37.4 & 800 & 120 & 110 & 437.5 & 1380 & 11900 & 25.4 & 120 & 14.5 & 72 & \multicolumn{2}{|c|}{80} \\
\hline$A_{\text {OLDC }}(\mathrm{dB})$ & 69 & - & 43 & 70 & $63.4^{\mathrm{b}}$ & 68 & 79 & 57.5 & $81.7^{\mathrm{b}}$ & 67 & 77 & \multicolumn{2}{|c|}{90.8} \\
\hline$C_{L}(p F)$ & 25 & 5 & 80 & $>5.5$ & 25 & 30 & 200 & 20 & 70 & 23 & 25 & $\begin{array}{c}\text { Small } \\
\mathrm{C}_{\mathrm{L}}= \\
25 \mathrm{pF}\end{array}$ & $\begin{array}{c}\text { Large } \\
C_{L}= \\
10 \mathrm{nF}\end{array}$ \\
\hline $\mathrm{SR}^{+}(\mathrm{V} / \mu \mathrm{s})$ & 0.77 & 11 & 100 & 19.5 & 2.7 & 22 & 74.1 & 1.8 & 9.8 & 24.11 & 43.1 & 31 & 0.107 \\
\hline$S_{R}^{-}(V / \mu s)$ & 0.31 & - & 78 & - & 3.3 & 24 & - & 3.8 & 7.6 & 23.33 & - & 28 & 0.105 \\
\hline$I_{\text {outMAX }}(\mu \mathrm{A})$ & 11 & 55 & - & 107.25 & 1120 & 660 & 14820 & - & 685.2 & 536.6 & 1077 & 980 & 1045 \\
\hline $\mathrm{CE}(\boldsymbol{\mu} \mathbf{A} / \mu \mathrm{A})$ & 0.53 & 0.34 & 104 & 1.17 & 6.4 & 1.58 & 2.24 & - & 11.42 & 67.08 & 27 & 22.8 & 24.3 \\
\hline GB (MHz) & 1.1 & $20^{\mathrm{c}}$ & 0.725 & 35 & $4.9^{\mathrm{b}}$ & 21.8 & 86.5 & 3 & 4.75 & 0.57 & 2.65 & $12.5^{\mathrm{c}}$ & $12.5^{\mathrm{c}}$ \\
\hline $\mathbf{P M}\left({ }^{\circ}\right)$ & 64 & - & 89.5 & $>45^{\mathrm{a}}$ & $83^{\mathrm{b}}$ & 73 & 50 & 60 & $60^{\mathrm{b}}$ & 82 & 86 & $58.4^{\mathrm{b}}$ & $78^{\mathrm{b}}$ \\
\hline $\mathrm{FOM}_{\mathrm{L}}(\boldsymbol{\mathrm { A }} \mathrm{A} / \mu \mathrm{W})$ & 0.29 & 0.07 & 59.33 & 0.98 & 2.56 & 0.48 & 1.25 & 2.2 & 4.43 & 37.3 & 15 & 12.66 & 13.06 \\
\hline $\mathrm{FOM}_{\mathrm{S}}(\mathrm{MHz} \cdot \mathrm{pF} / \mu \mathrm{W})$ & 0.74 & 0.13 & 0.48 & 1.75 & 0.28 & 0.47 & 1.46 & 2.36 & 2.77 & 0.9 & 0.92 & $3.90^{\mathrm{f}}$ & $2.25^{\mathrm{f}}$ \\
\hline$\frac{\text { FOM }_{\mathrm{G}}}{(\sqrt{\mu \mathrm{MMHzpF}} / \mu \mathrm{W})}$ & 0.46 & 0.1 & 5.34 & 1.31 & 0.85 & 0.475 & 1.35 & 2.28 & 3.5 & 5.8 & 3.71 & 7.03 & 5.42 \\
\hline Offset (mV) & 6.18 & - & - & - & - & 0.2 & - & 11 & 6 & - & - & \multicolumn{2}{|c|}{2.48} \\
\hline Input Noise $(\mathrm{nV} / \sqrt{H z})$ & 25 & - & 230 & 14.14 & - & - & 0.8 & 100 & 35 & - & - & \multicolumn{2}{|c|}{27} \\
\hline CMRR (dB) & 72 & - & 68 & - & $80^{\mathrm{b}}$ & 75 & - & 19 & 78 & 73.2 & - & \multicolumn{2}{|c|}{68} \\
\hline PSRR $+(d B)$ & 76 & - & 55 & - & 61.2 & 78 & - & 52.1 & 72 & 44.1 & - & \multicolumn{2}{|c|}{64} \\
\hline PSRR- (dB) & 70 & - & 58 & - & - & 75 & - & 66.4 & 74 & 41.8 & - & \multicolumn{2}{|c|}{66} \\
\hline THD $^{\mathrm{d}}(\%$ or $\mathrm{dB})$ & $-42^{\mathrm{e}}$ & - & -56 & - & $-47.1^{b}$ & -54 & - & $<\% 1$ & -41 & -48 & - & \multicolumn{2}{|c|}{-50} \\
\hline ICMR (V) & 1.35 & - & - & - & - & 2.65 & - & 0.55 & - & 1.28 & - & \multicolumn{2}{|c|}{1.35} \\
\hline Area $\left(\mathrm{mm}^{2}\right)$ & 0.019 & 0.045 & 0.024 & 0.012 & 0.029 & 0.034 & 0.070 & 0.0198 & 0.024 & 0.030 & 0.007 & \multicolumn{2}{|c|}{0.021} \\
\hline
\end{tabular}

${ }^{a}$ Assuming closed-loop gain of 2

${ }^{\mathrm{b}}$ Simulated

${ }^{\mathrm{c}}$ Unity gain frequency $f_{U G}$

d @ $100 \mathrm{kHz}, 1-\mathrm{Vp}-\mathrm{p}$ input signal

'@ $100 \mathrm{kHz}, 0.1-\mathrm{Vp}-\mathrm{p}$ input signal

${ }^{\mathrm{f}}$ The $B W$ used for FOMs is measured at $V_{\text {out }}$,

On the other hand, robustness of $P M$ (stability) is checked by performing 1000 Monte Carlo runs including the process and mismatch data. The capacitive load in these simulations is considered $C_{L}=25 \mathrm{pF}$. The result of these simulations is illustrated in Fig. 9.

The simulated open-loop response (magnitude $\left|A_{O L}\right|$ and phase) of the proposed (with $R_{S}=900 \Omega$ ) and conventional op-amps of Figs. 1 and 2(b), respectively, are illustrated in Fig. 10(a) for two extreme values of capacitive loads $C_{L}=25 \mathrm{pF}$ and $C_{L}=10 \mathrm{nF}$. A low value $C_{L}=25 \mathrm{pF}$ is used for simulations since it corresponds to the parasitic load capacitance in the chip test setup. The conventional class-A Miller op-amp has the same parameters $\left(W / L, R_{z}, I_{\text {bias }}\right.$, etc.) as the proposed op-amp with exception of $C_{c}$ that has a value $C_{c}=15 \mathrm{pF}$.

It can be seen that in the proposed class-AB-AB op-amp $\left|A_{O L D C}\right|=91.2 \mathrm{~dB}$, as expected, equal values of unity-gain frequency $f_{U G} \approx 12 \mathrm{MHz}$ are obtained for both capacitive loads. This validates the discussion in Section II-B. In addition, nominal $C_{L}=25 \mathrm{pF}$ and large $C_{L}=10 \mathrm{nF}$ result in $P M \approx 60^{\circ}$ and $P M \approx 80^{\circ}$, respectively, in the proposed circuit. The simulated $P M \mathrm{~s}$ can be validated using values in Table III and (15). On the other hand, the conventional op-amp has $\left|A_{O L D C}\right|=70 \mathrm{~dB}$. The phase margin is $P M \approx$ $66^{\circ}$ for nominal $C_{L}=25 \mathrm{pF}$ and only $P M \approx 21^{\circ}$ for large $C_{L}=10 \mathrm{nF}$. In addition, the simulated open-loop (ac) response of the proposed op-amp and the amplifiers in [1] and [4] are shown and compared in Fig. 10(b) and (c), respectively. Furthermore, the large-signal transient response of the proposed op-amp is simulated and compared in Fig. 11 for two $C_{L}$ loads; $C_{L}=25 \mathrm{pF}$ [Fig. 11(a)] and $C_{L}=10 \mathrm{nF}$ [Fig. 11(b)]. For transient behavior, the amplifier was measured in voltage follower configuration (node $V_{\text {out }}$ connected to the negative input terminal) and the output $\left(V_{\text {out }}{ }^{\prime}\right)$ was considered after $R_{S}$. For a fair comparison, all amplifiers are simulated in 180-nm CMOS technology with the same parameters $(W / L$, $C_{c}, R_{z}, R_{s}, I_{\text {bias }}$, etc). Fig. 10 (b) validates that for any $C_{L}$, the proposed class-AB-AB op-amp has higher $\left|A_{O L D C}\right|, f_{U G}$, $S R, I_{\text {out } M A X}$, and $P M$ than the scheme in [1] $\left[f_{U G[1]}=\right.$ $\left.g_{m D P} /\left(\pi C_{c}\right)\right]$.

On the other hand, Fig. 10(c) shows that for different $C_{L}$ loads, $f_{U G}$ of the proposed op-amp $\left(f_{U G}=\right.$ $\left.\left(\left(g_{m e f f} g_{m I I} R_{S}\right) /\left(2 \pi C_{c}\left(1+g_{m I I} R_{S}\right)\right)\right)\right)$ remains constant while in [4] $f_{U G[4]}=g_{m e f f} /\left(2 \pi C_{L}\right)$ highly decreases for 
$C_{L}=10 \mathrm{nF}$. Note that in [4], for $C_{L}=10 \mathrm{nF}$, the zero $f_{z}=1 /$ $\left(2 \pi R_{S} C_{L}\right)$ moves below the constant nondominant pole $f_{p A}=1 /\left(2 \pi R_{A} C_{A}\right)$ and the $C_{L}$ dependent pole-zero cancelation is disturbed. This movement increases $P M$; however, based on discussion made before, the settling time increases. This is demonstrated in Fig. 11(b). The transient results of Fig. 11 show that for any $C_{L}$ loads, $S R(C E)$ and the settling time of the proposed class-AB-AB op-amp are better than those of the conventional Miller op-amp and the schemes in [1] and [4].

The robustness of the proposed circuit of Fig. 2(b) with $C_{L}=25 \mathrm{pF}, R_{S}=900 \Omega, V_{D D}=0.9 \mathrm{~V}, V_{S S}=-0.9 \mathrm{~V}$, and $I_{\text {bias }}=5 \mu \mathrm{A}$ was simulated over process corners (TT, SS, FF, SF, and FS) and temperature variations $\left(T=-40{ }^{\circ} \mathrm{C}, 27^{\circ} \mathrm{C}\right.$, and $\left.125^{\circ} \mathrm{C}\right)$. The results are summarized in Table V. It can be seen that the performance of the proposed op-amp shows relatively small changes with process and temperature variations.

\section{B. Measurement Results}

Fig. 12 shows the micrograph of the test chip including the proposed class-AB-AB op-amp [Fig. 2(b)] and a conventional Miller op-amp (Fig. 1) fabricated in the same 180-nm CMOS technology used for the simulations. Since the opaque passivation hides the active devices, the layouts of op-amps are superimposed in Fig. 12. The dimensions of the conventional and proposed circuits are $194 \mu \mathrm{m} \times 97 \mu \mathrm{m}\left(0.019 \mathrm{~mm}^{2}\right)$ and $212 \times 97 \mu \mathrm{m}\left(0.021 \mathrm{~mm}^{2}\right)$, respectively.

In both proposed and conventional op-amps, the same parameters (W/L, $R_{S}, V_{D D}, V_{S S}, I_{\text {bias }}$, etc.) as in simulations were used for fabrication and testing. The measured transient responses of both op-amps in voltage follower configuration to two different $1.35-\mathrm{V}_{\mathrm{p}-\mathrm{p}}$ and $0.1-\mathrm{V}_{\mathrm{p}-\mathrm{p}}$ input square waveforms are depicted in Fig. 13. Two extreme values of $C_{L}=25 \mathrm{pF}$ (Fig. 13, left waveforms) and $C_{L}=10 \mathrm{nF}$ (Fig. 13, right waveforms) are used for this purpose. Table VI summarizes the performance parameters of the proposed op-amp [experimental results of Fig. 2(b) for $C_{L}=25 \mathrm{pF}$ and $\left.C_{L}=10 \mathrm{nF}\right]$ and compares it to other recent class- $\mathrm{AB}$ schemes as well as to the conventional Miller op-amp (Fig. 1). Note that in the proposed circuit, the mismatches for each element (i.e., $C_{L}, R_{S}, R, \ldots$ ) are determined by manufacturing tolerances of $\sim 20 \%$. Table VI also includes the input-referred noise density $(\mathrm{nV} / \sqrt{ } \mathrm{Hz})$ at $1 \mathrm{MHz}$ and also the total harmonic distortion of the output voltage of the two proposed class- $\mathrm{AB}$ op-amps in voltage follower configuration for $1-\mathrm{V}_{\mathrm{p}-\mathrm{p}}$ input at $100-\mathrm{kHz}$ frequency and for the conventional class-A Miller op-amp for $0.1-\mathrm{V}_{\mathrm{p}-\mathrm{p}}$ input at $100-\mathrm{kHz}$ frequency. It is noticeable that Fig. 2(b) circuit has essential performance improvements in most of the parameters especially $C E, F O M_{L}$, $\mathrm{FOM}_{S}$, and $\mathrm{FOM}_{G}$.

\section{CONCLUSION}

A simple power-efficient pseudo two-stage class-AB-AB Miller op-amp that is stable over a wide range of capacitive loads starting at $C_{\text {Lmin }}=15 \mathrm{pF}$ is introduced. Stability is enforced using a combination of Miller and phase-lead compensations as well as $C_{L}$-independent pole-zero cancelation. This provides stability, a constant $f_{U G}$, and approximately constant settling time over a wide range of $C_{L}$ loads. Its class-AB-AB operation is based on combination of $g_{m}$ boosting technique and a nonlinear load to achieve high output current enhancement factors. The $g_{m}$ boosting also provides enhanced $B W$ and gain. The proposed scheme of Fig. 2(b) was experimentally verified with a test chip prototype fabricated in 180-nm CMOS technology. The proposed class-AB-AB op-amp showed higher performance in terms of $A_{O L D C}, C E$, $F O M_{G}$ (both $F O M s$, and $F O M_{L}$ ), compared to other class$\mathrm{AB}$ schemes reported in the literature.

\section{REFERENCES}

[1] J. Aguado-Ruiz, A. Lopez-Martin, J. Lopez-Lemus, and J. RamirezAngulo, "Power efficient class $\mathrm{AB}$ op-amps with high and symmetrical slew rate," IEEE Trans. Very Large Scale Integr. (VLSI) Syst., vol. 22, no. 4, pp. 943-947, Apr. 2014.

[2] M. Figueiredo, R. Santos-Tavares, E. Santin, J. Ferreira, G. Evans, and J. Goes, "A two-stage fully differential inverter-based self-biased CMOS amplifier with high efficiency," IEEE Trans. Circuits Syst. I, Reg. Papers, vol. 58, no. 7, pp. 1591-1603, Jul. 2011.

[3] E. Cabrera-Bernal, S. Pennisi, A. D. Grasso, A. Torralba, and R. G. Carvajal, " $0.7-\mathrm{V}$ three-stage class-AB CMOS operational transconductance amplifier," IEEE Trans. Circuits Syst. I, Reg. Papers, vol. 63, no. 11, pp. 1807-1815, Nov. 2016.

[4] J. Ramirez-Angulo and M. Holmes, "Simple technique using local CMFB to enhance slew rate and bandwidth of one-stage CMOS opamps," Electron. Lett., vol. 38, no. 23, pp. 1409-1411, Nov. 2002.

[5] P. R. Surkanti and P. M. Furth, "Converting a three-stage pseudoclass$\mathrm{AB}$ amplifier to a true-class-AB amplifier," IEEE Trans. Circuits Syst. II, Exp. Briefs, vol. 59, no. 4, pp. 229-233, Apr. 2012.

[6] A. J. Lopez-Martin, S. Baswa, J. Ramirez-Angulo, and R. G. Carvajal, "Low-voltage super class AB CMOS OTA cells with very high slew rate and power efficiency," IEEE J. Solid-State Circuits, vol. 40, no. 5, pp. 1068-1077, May 2005.

[7] S. Sutula, M. Dei, L. Terés, and F. Serra-Graells, "Variable-mirror amplifier: A new family of process-independent class-AB single-stage OTAs for low-power SC circuits," IEEE Trans. Circuits Syst. I, Reg. Papers, vol. 63, no. 8, pp. 1101-1110, Aug. 2016.

[8] A. Lopez-Martin, M. P. Garde, J. M. Algueta, A. Carlos, R. G. Carvajal, and J. Ramirez-Angulo, "Enhanced single-stage folded cascode OTA suitable for large capacitive loads," IEEE Trans. Circuits Syst. II, Exp. Briefs, vol. 65, no. 4, pp. 441-445, Apr. 2018.

[9] S. Pourashraf, J. Ramirez-Angulo, A. J. Lopez-Martin, and R. G. Carvajal, "Super class AB OTA without open-loop gain degradation based on dynamic cascode biasing," Int. J. Circuit Theory Appl., vol. 45, no. 12, pp. 2111-2118, Dec. 2017.

[10] S. Pourashraf et al., "High current efficiency class-AB OTA with high open loop gain and enhanced bandwidth," IEICE Electron. Exp. Lett., vol. 14, no. 17, p. 20170719 , Aug. 2017.

[11] F. Dielacher, J. Hauptmann, J. Reisinger, R. Steiner, and H. Zojer, "A software programmable CMOS telephone circuit," IEEE J. Solid-State Circuits, vol. 26, no. 7, pp. 1015-1026, Jul. 1991.

[12] B. J. Blalock, P. E. Allen, and G. A. Rincon-Mora, "Designing 1-V op amps using standard digital CMOS technology," IEEE Trans. Circuits Syst. II, Analog Digit. Signal Process., vol. 45, no. 7, pp. 769-780, Jul. 1998.

[13] A. Guzinski, M. Bialko, and J. C. Matheau, "Body driven differential amplifier for application in continuous-time active-C filter," in Proc. Eur. Conf. Circuit Theory Design, Jun. 1987, pp. 315-319.

[14] W. Sansen, "Class AB and driver amplifiers," in Analog Design Essentials, W. Sansen, Ed., 1st ed. New York, NY, USA: Springer-Verlag, 2006, pp. 337-362.

[15] J. Ramirez-Angulo, R. G. Carvajal, J. A. Galan, and A. LopezMartin, "A free but efficient low-voltage class-AB two-stage operational amplifier," IEEE Trans. Circuits Syst. I: Reg. Papers, vol. 53, no. 7, pp. 568-571, Jul. 2006.

[16] W. M. Sansen, "Class AB and driver amplifiers," in Analog Design Essentials. New York, NY, USA: Springer-Verlag, 2007. 
This is the author's version of an article that has been published in this journal. Changes were made to this version by the publisher prior to publication. The final version of record is available at https://doi.org/10.1109/TVLSI.2018.2830365

[17] J. A. Galan, A. J. Lopez-Martin, R. G. Carvajal, J. Ramirez-Angulo, and C. Rubia-Marcos, "Super class-AB OTAs with adaptive biasing and dynamic output current scaling," IEEE Trans. Circuits Syst. I, Reg. Papers, vol. 54, no. 3, pp. 449-457, Mar. 2007.

[18] A. D. Grasso, D. Marano, F. Esparza-Alfaro, A. J. Lopez-Martin, G. Palumbo, and S. Pennisi, "Self-biased dual-path push-pull output buffer amplifier for LCD column drivers," IEEE Trans. Circuits Syst. I, Reg. Papers, vol. 61, no. 3, pp. 663-670, Mar. 2014.

[19] A. D. Grasso, D. Marano, G. Palumbo, and S. Pennisi, "Design methodology of subthreshold three-stage CMOS OTAs suitable for ultra-low-power low-area and high driving capability," IEEE Trans. Circuits Syst. I, Reg. Papers, vol. 62, no. 6, pp. 1453-1462, Jun. 2015.

[20] A. D. Grasso, D. Marano, G. Palumbo, and S. Pennisi, "Highperformance three-stage single-miller CMOS OTA with no upper limit of CL," IEEE Trans. Circuits Syst. II, Exp. Brief, to be published, doi: 10.1109/TCSII.2017.2756923.

[21] W. Qu, S. Singh, Y. Lee, Y. S. Son, and G. H. Cho, "Designoriented analysis for miller compensation and its application to multistage amplifier design," IEEE J. Solid-State Circuits, vol. 52, no. 2, pp. 517-527, Feb. 2017.

[22] M. Tan and W.-H. Ki, "A cascode Miller-compensated three-stage amplifier with local impedance attenuation for optimized complex-pole control," IEEE J. Solid-State Circuits, vol. 50, no. 2, pp. 440-449, Feb. 2015.

[23] T. Stockstad and H. Yoshizawa, "A 0.9-V $0.5 \mu \mathrm{m}$ rail-to-rail CMOS operational amplifier," IEEE J. Solid-State Circuits, vol. 37, no. 3, pp. 286-292, Mar. 2002.

[24] A. D. Grasso, P. Monsurró, S. Pennisi, G. Scotti, and A. Trifilietti, "Analysis and implementation of a minimum-supply body-biased CMOS differential amplifier cell," IEEE Trans. Very Large Scale Integr. (VLSI) Syst., vol. 17, no. 2, pp. 172-180, Feb. 2009.

[25] P. Monsurró, G. Scotti, A. Trifiletti, and S. Pennisi, "Biasing technique via bulk terminal for minimum supply CMOS amplifiers," Electron. Lett., vol. 41, no. 14, pp. 779-780, Jul. 2005.

[26] M. Herpy, Analog Integrated Circuits: Operational Amplifiers and Analog Multipliers. Hoboken, NJ, USA: Wiley, 1980.

[27] S. Franco. (Aug. 27, 2013). Demystifying Pole-Zero Doublets. Accessed: Jan. 17, 2018. [Online]. Available: https://www.edn.com/electronicsblogs/analog-bytes/4420171/Demystifying-pole-zero-doublets-

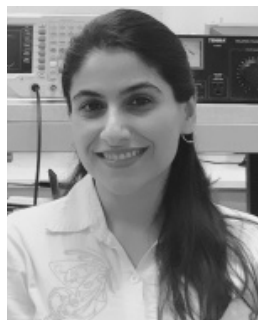

Shirin Pourashraf (M'10) was born in Darreh Shahr, Ilam, Iran, in 1984. She received the M.S. degree in microelectronics and digital VLSI design from the Isfahan University of Technology, Esfahan, Iran, in 2011. She is currently working toward the $\mathrm{Ph} . \mathrm{D}$. degree at the Klipsch School of Electrical and Computer Engineering Department, New Mexico State University, Las Cruces, NM, USA.

Since 2013, she has been with the Klipsch School of Electrical and Computer Engineering Department, New Mexico State University, where she is currently an Assistant Researcher. Her current research interests include design and test of low-voltage/low-power and high-performance analog-/mixed-signal building blocks.

Ms. Pourashraf was awarded as "Outstanding Teaching Assistant" in the Klipsch School of Electrical and Computer Engineering Department.

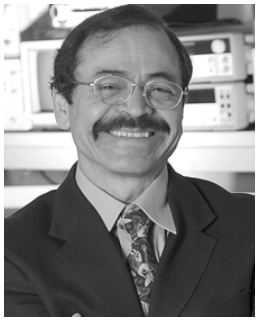

Jaime Ramirez-Angulo (F'10) received the B.Sc. degree in communications and electronic engineering and the M.S.E.E. degree from the National Polytechnic Institute, Mexico City, Mexico, in 1974 and 1976, respectively, and the Dr.-Ing. degree from the University of Stuttgart, Stuttgart, Germany, in 1982.

$\mathrm{He}$ was a Professor at the National Institute for Astrophysics Optics and Electronics, Cholula, Mexico, and with Texas A\&M University, College Station, TX, USA. He is currently a Distinguished Award Professor at the Klipsch School of Electrical and Computer Engineering, New Mexico State University, Las Cruces, NM, USA, where he is also the Director of the Mixed-Signal VLSI Laboratory. His current research interests include various aspects of design and test of analog- and mixed-signal very-large-scale integrated circuits.

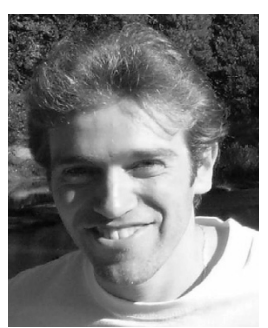

Antonio J. Lopez-Martin (M'04-SM'11) received the M.S. and Ph.D. (honors) degrees from the Public University of Navarra, Pamplona, Spain, in 1995 and 1999 , respectively.

He was a Visiting Professor at New Mexico State University, Las Cruces, NM, USA, and an Invited Researcher at the Swiss Federal Institute of Technology, Zurich, Switzerland. He is currently a Professor at the Public University of Navarra, and an Adjunct Professor at New Mexico State University. He is a consultant for local companies. He has authored over 400 technical contributions in books, journals, and conferences, and holds six international patents. His current research interests include wireless transceivers and sensor interfaces with emphasis on low-voltage low-power implementations.

Dr. Lopez-Martin is with the technical committee of various conferences. He was a recipient of the Talgo Technological Innovation Award in 2012, the ANIT's Engineer of the Year Award in 2008, the Caja Navarra Research Award in 2007, the Young Investigator Award from the Complutense University of Madrid in 2006, the 2005 IEEE TRANSACTIONS ON EDUCATION Best Paper Award, and the European Center of Industry and Innovation Award in 2004 for excellence in transfer of research results to industry. $\mathrm{He}$ was an Associate Editor of the IEEE TRANSACTIONS ON Circuits AND SYSTEMS-II: EXPRESS BRIEFS from 2006 to 2007 and the IEEE TRANSACTIONS ON Circuits AND Systems-I: Regular PaPers from 2008 to 2009.

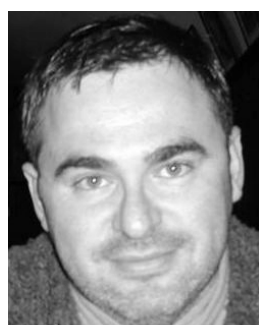

Ramon González-Carvajal (M'99-SM'04) was born in Seville, Spain. He received the Electrical Engineering and Ph.D. (honors) degrees from the University of Seville, Seville, Spain, in 1995 and 1999, respectively.

He joined the Department of Electronic Engineering, School of Engineering, University of Seville, as an Associate Professor, in 1996, where he became a Professor in 2002. He joined the Electrical Engineering Department, Texas A\&M University, College Station, TX, USA, as an Invited Researcher, in 1997. He joined the Klipsch School of Electrical Engineering, NMSU, Las Cruces, NM, USA, as an Invited Researcher, in 1999. He was an Invited Researcher with the Klipsch School of Electrical Engineering, NMSU, from 2001 to 2004, where he is also an Adjunct Professor. He has authored over 60 papers in international journals and over 130 in international conferences. His current research interests include low-voltage low-power analog circuit design, A/D and D/A conversion, and analog- and mixed-signal processing. 